\title{
High-quality axions in solutions to the $\mu$ problem
}

\author{
Prudhvi N. Bhattiprolu॰ and Stephen P. Martin® \\ Department of Physics, Northern Illinois University, DeKalb, Illinois 60115, USA
}

(Received 8 July 2021; accepted 10 August 2021; published 13 September 2021)

\begin{abstract}
Solutions to the $\mu$ problem in supersymmetry based on the Kim-Nilles mechanism naturally feature a Dine-Fischler-Srednicki-Zhitnitsky (DFSZ) axion with a decay constant of the order of the geometric mean of the Planck and $\mathrm{TeV}$ scales, consistent with astrophysical limits. We investigate minimal models of this type with two gauge-singlet fields that break a Peccei-Quinn symmetry and extensions with an extra vectorlike quark and lepton supermultiplets consistent with gauge coupling unification. We show that there are many anomaly-free discrete symmetries, depending on the vectorlike matter content, that protect the Peccei-Quinn symmetry to sufficiently high order to solve the strong $C P$ problem. We study the axion couplings in this class of models. Models of this type that are automatically free of the domain wall problem require at least one pair of strongly interacting vectorlike multiplets with mass at the intermediate scale and predict axion couplings that are greatly enhanced compared to the minimal supersymmetric DFSZ models, putting them within reach of proposed axion searches.
\end{abstract}

DOI: 10.1103/PhysRevD.104.055014

\section{INTRODUCTION}

Extensions of the Standard Model (SM) of particle physics are plagued by several apparent hierarchy problems, which can be viewed as hints toward the ultimate completion of the theory. The vacuum energy, as manifested in the observed expansion rate of the Universe due to the cosmological constant, is approximately 120 orders of magnitude smaller than its naive dimensional analysis estimate $M_{P}^{4}$, where $M_{P}=2.4 \times 10^{18} \mathrm{GeV}$ is the reduced Planck mass scale. The "big hierarchy problem" is that the electroweak scale set by the Higgs field squared mass parameter is 32 orders of magnitude smaller than $M_{P}^{2}$. The strong $C P$ problem is that the $C P$-odd angle $\theta$ in the QCD Lagrangian could be of the order of 1 but is constrained to be smaller than $9 \times 10^{-11}$ by the measured value [1] of the electric dipole moment of the neutron.

In this paper, we will be concerned with a class of models that simultaneously address the latter two problems. The big hierarchy problem of the Higgs field is addressed by supersymmetry, with supersymmetry-breaking terms

Published by the American Physical Society under the terms of the Creative Commons Attribution 4.0 International license. Further distribution of this work must maintain attribution to the author(s) and the published article's title, journal citation, and DOI. Funded by SCOAP ${ }^{3}$. characterized by the $\mathrm{TeV}$ scale, ${ }^{1}$ while the strong $C P$ problem is addressed by including a global Peccei-Quinn (PQ) $U(1)$ symmetry $[2,3]$ that is explicitly broken by a QCD anomaly but also spontaneously broken, minimizing the effective value of $|\theta|$ and giving rise to a light, very weakly coupled axion [4-12]. For reviews of the axion solution to the strong $C P$ problem from various points of view, see Refs. [13-22].

The " $\mu$ problem" of supersymmetry relates the issues of supersymmetry breaking and PQ symmetry breaking. The superpotential of the minimal supersymmetric Standard Model (MSSM; for a review, see [23]) contains terms of the form

$$
W=\mu H_{u} H_{d}+y_{u} H_{u} q \bar{u}-y_{d} H_{d} q \bar{d}-y_{e} H_{d} \ell \bar{e},
$$

where $H_{u}$ and $H_{d}$ are the Higgs doublet chiral superfields. The $q, \bar{u}, \bar{d}, \ell$, and $\bar{e}$ are quark and lepton chiral superfields, for which we suppress flavor and gauge indices and use lowercase letters to distinguish them from additional

\footnotetext{
${ }^{1}$ In this paper, we will loosely refer to the mass scales associated with superpartner masses and supersymmetry breaking as the $\mathrm{TeV}$ scale. Given the negative search results so far at the Large Hadron Collider (LHC), the masses of the strongly interacting superpartners are evidently somewhat larger than $1 \mathrm{TeV}$. The fact that their masses exceed the Higgs vacuum expectation value by an order of magnitude is the "little hierarchy problem," which we do not address in this paper; it is obviously much less severe than the big hierarchy problem, for which the LHC results have not favored any competing hypotheses.
} 
vectorlike quark and lepton superfields to be considered below. Besides the Yukawa coupling matrices $y_{u}, y_{d}$, and $y_{e}$, this superpotential contains a single dimensionful parameter $\mu$, which preserves supersymmetry but nevertheless should be roughly of the same order as the supersymmetry-breaking mass scale in order to allow for electroweak symmetry breaking. To accomplish this, the Kim-Nilles mechanism [24] takes the $\mu$ term to be absent from Eq. (1.1) in the ultraviolet theory and in its place introduces nonrenormalizable terms, for example, of the form proposed in Ref. [25]:

$$
W_{\mathrm{I}}=\frac{\lambda_{\mu}}{M_{P}} X Y H_{u} H_{d}+\frac{\lambda}{6 M_{P}} X^{3} Y,
$$

where $X$ and $Y$ are gauge-singlet chiral superfields and $\lambda_{\mu}$ and $\lambda$ are dimensionless couplings. The term $\lambda$ in Eq. (1.2) is included to stabilize the potential at large $X, Y$ scalar field strengths. Including the effects of supersymmetry breaking, there are also contributions to the Lagrangian (in terms of scalar fields, for which we use the same symbol as the corresponding superfield):

$$
\begin{aligned}
\mathcal{L}_{\text {soft }}= & \left(\frac{a_{\mu}}{M_{P}} X Y H_{u} H_{d}+\frac{a}{6 M_{P}} X^{3} Y\right)+\text { c.c. } \\
& -m_{X}^{2}|X|^{2}-m_{Y}^{2}|Y|^{2}
\end{aligned}
$$

where $a_{\mu}$ and $a$ are of the order of $m_{\text {soft }}$ and $m_{X}^{2}$ and $m_{Y}^{2}$ are of the order of $m_{\text {soft }}^{2}$, with $m_{\text {soft }}$ roughly at the TeV scale. If $m_{X}^{2}$ and $m_{Y}^{2}$ are negative, or just sufficiently small compared to $|a / \lambda|^{2}$, then the resulting scalar potential has a local minimum for vacuum expectation values (VEVs) parametrically of the order of ${ }^{2}$

$$
\langle X\rangle \sim\langle Y\rangle \sim \sqrt{m_{\mathrm{soft}} M_{P}} \equiv M_{\mathrm{int}},
$$

a scale intermediate between the Planck and $\mathrm{TeV}$ scales. This can always occur if $m_{X}^{2}$ and $m_{Y}^{2}$ are negative, but this is not a necessary condition. For example, if $m_{X}^{2}=$ $m_{Y}^{2}=m^{2}$, then there is a nontrivial local minimum if $|a / \lambda|^{2}-12 m^{2}>0$, and it will be a global minimum if $|a / \lambda|^{2}-16 m^{2}>0$. Symmetry breaking along such flat directions is stable against radiative corrections [26]. In the low-energy theory, the $\mu$ and $b$ terms of the MSSM Lagrangian are

$$
\mu=\frac{\lambda_{\mu}}{M_{P}}\langle X Y\rangle \sim m_{\mathrm{soft}},
$$

\footnotetext{
${ }^{2}$ Note that it is important to include the contribution of the allowed holomorphic supersymmetry-breaking coupling $a$ here. Omitting it would seemingly lead to $\langle Y\rangle \ll\langle X\rangle \sim M_{\text {int }}$.
}

$$
b=\frac{a_{\mu}}{M_{P}}\langle X Y\rangle \sim m_{\mathrm{soft}}^{2}
$$

respectively, solving the $\mu$ problem.

If $m_{\text {soft }}$ is of the order of the TeV scale, then

$$
10^{9} \mathrm{GeV} \lesssim M_{\text {int }} \lesssim 10^{12} \mathrm{GeV},
$$

depending on the dimensionless parameters involved. Equation (1.7) roughly coincides with the preferred range for the decay constant of the axion from astrophysical constraints on the low end and from cosmological dark matter density on the upper end. It is, therefore, notable that $X$ and $Y$ carry nonzero charges for a PQ symmetry, which the VEVs spontaneously break, so that the axion could be a linear combination of the pseudoscalar components of the fields $X$ and $Y$ (with a very small admixture of $H_{u}$ and $H_{d}$ ). Since the MSSM quarks also carry nonzero PQ charge, this is an example of a Dine-Fischler-Srednicki-Zhitnitsky (DFSZ) [8,9] axion model. In this way, the solution of the $\mu$ problem in supersymmetry can also be the solution of the strong $C P$ problem.

Besides the axion, the other components of the supermultiplets $X$ and $Y$ all get TeV-scale masses. These include a scalar saxion, and gauge-singlet axino fermions, one of which could be the lightest supersymmetric particle. The lightest MSSM superpartner could decay to the axino with a macroscopic proper decay length, which can be much larger than the size of a collider detector. However, some of the decays can occur within the detector, providing a striking search signal [27] for the Large Hadron Collider.

In addition to Eq. (1.2), there are three other similar but distinct superpotential structures involving two PQ-breaking superfields $X$ and $Y$. (For simplicity and economy, we restrict our attention to only two such fields. Although it is possible to have more than two, this would seem to make it harder to find solutions to the axion quality problem to be discussed shortly.) They are

$$
\begin{gathered}
W_{\mathrm{II}}=\frac{\lambda_{\mu}}{2 M_{P}} X^{2} H_{u} H_{d}+\frac{\lambda}{6 M_{P}} X^{3} Y, \\
W_{\mathrm{III}}=\frac{\lambda_{\mu}}{2 M_{P}} Y^{2} H_{u} H_{d}+\frac{\lambda}{6 M_{P}} X^{3} Y, \\
W_{\mathrm{IV}}=\frac{\lambda_{\mu}}{2 M_{P}} X^{2} H_{u} H_{d}+\frac{\lambda}{4 M_{P}} X^{2} Y^{2},
\end{gathered}
$$

with corresponding holomorphic soft supersymmetrybreaking parameters $a_{\mu}$ and $a$ in each case, in the obvious way. The structure in Eq. (1.8) was proposed in Ref. [28], and those in Eqs. (1.9) and (1.10) in Ref. [27]. For a review and further elucidation, see [29]. In the following, we will refer to the models defined by Eqs. (1.2), (1.8), (1.9), and (1.10) as base models $\mathrm{B}_{\mathrm{I}}, \mathrm{B}_{\mathrm{II}}, \mathrm{B}_{\mathrm{III}}$, and $\mathrm{B}_{\mathrm{IV}}$, respectively, 
TABLE I. The four base models. Each model has two gaugesinglet chiral superfields $X$ and $Y$ whose scalar component VEVs spontaneously break the Peccei-Quinn symmetry and generate the $\mu$ term through the schematic superpotential terms shown. The PQ charges are normalized so that $H_{u} H_{d}$ has charge -2 .

\begin{tabular}{lcc}
\hline \hline Base model & Superpotential terms & PQ charges of $(X, Y)$ \\
\hline $\mathrm{B}_{\mathrm{I}}$ & $X Y H_{u} H_{d}+X^{3} Y$ & $(-1,3)$ \\
$\mathrm{B}_{\mathrm{II}}$ & $X^{2} H_{u} H_{d}+X^{3} Y$ & $(1,-3)$ \\
$\mathrm{B}_{\text {III }}$ & $Y^{2} H_{u} H_{d}+X^{3} Y$ & $(-1 / 3,1)$ \\
$\mathrm{B}_{\mathrm{IV}}$ & $X^{2} H_{u} H_{d}+X^{2} Y^{2}$ & $(1,-1)$ \\
\hline \hline
\end{tabular}

since we will be interested in extensions of them. Each of them implies a different assignment of PQ charges, which are summarized in Table I. We choose the normalization of the PQ charges so that $H_{u} H_{d}$ has charge -2 .

The axion quality problem [30-34] results from the possible presence of higher-dimensional contributions to the Lagrangian that explicitly violate the $U(1) \mathrm{PQ}$ symmetry, since these can displace the QCD $\theta$ parameter away from 0 at the minimum of the scalar potential, spoiling the solution to the strong $C P$ problem. Such contributions are expected to be allowed if they are not forbidden, because ungauged symmetries are not respected by quantum gravitational effects, at least in thought experiments such as black hole evaporation processes. In supersymmetry, we therefore consider superpotential operators of the form

$$
W=\frac{\kappa}{M_{P}^{p-3}} X^{j} Y^{p-j}
$$

with dimensionless $\kappa$. Together with the $\lambda$ term in the superpotential, this gives rise to scalar potential terms (from $\left|F_{X}\right|^{2}$ and $\left|F_{Y}\right|^{2}$ ) that have $p+2$ powers of $X$ and $Y$ and are suppressed by $1 / M_{P}^{p-2}$. These are also accompanied by holomorphic supersymmetry-breaking terms in the scalar potential

$$
V=\frac{a_{\kappa}}{M_{P}^{p-3}} X^{j} Y^{p-j}+\text { c.c. }
$$

where $a_{\kappa}$ should be of the order of the TeV scale. For generic phases of $\kappa$ and $a_{\kappa}$, both of these types of contributions result in tadpoles for the axion field $A$ (a linear combination of the imaginary parts of $X$ and $Y$ expanded around the $C P$-conserving vacuum) that are parametrically of the same form:

$$
V=-\delta \frac{f_{A}^{p+1}}{M_{P}^{p-2}} A,
$$

where we have identified the axion decay constant $f_{A}$ with the intermediate-scale VEVs $M_{\text {int }}$ and the dimensionless quantity $\delta$ depends on the parameters (including the magnitudes and phases of $\lambda, \kappa$, and $a_{\kappa}$ and the integer $k$ ) in a complicated way. The axion potential also includes the usual squared mass term, approximately for small $A$,

$$
V=\frac{\mathcal{M}_{\mathrm{QCD}}^{4}}{2 f_{A}^{2}} A^{2},
$$

where

$$
\mathcal{M}_{\mathrm{QCD}}^{4} \approx \frac{m_{u} m_{d} m_{\pi}^{2} f_{\pi}^{2}}{\left(m_{u}+m_{d}\right)^{2}} \approx(0.0754 \mathrm{GeV})^{4}
$$

The combination of Eqs. (1.13) and (1.14) gives rise to

$$
\left|\theta_{\mathrm{eff}}\right|=\langle A\rangle / f_{A}=\delta \frac{f_{A}^{p+2}}{\mathcal{M}_{\mathrm{QCD}}^{4} M_{P}^{p-2}} .
$$

Now by requiring that the $\theta_{\text {eff }}$ parameter at the minimum of the potential is less than $10^{-10}$ from the experimental bound on the neutron electric dipole moment, one therefore finds [32-34] that one should have

$$
p+2>\frac{88+\log _{10}(\delta)}{9.4-\log _{10}\left(f_{A} / 10^{9} \mathrm{GeV}\right)} .
$$

Note that the bound on $p$ becomes weaker for smaller $f_{A}$. With the naive $\delta \approx 1$, if PQ-violating superpotential terms with $p=(8,9,10,11$, or 12$)$ are present, one should have $f_{A} \lesssim\left(4 \times 10^{9}, 3 \times 10^{10}, 10^{11}, 4 \times 10^{11}\right.$, or $\left.10^{12}\right) \mathrm{GeV}$, respectively. However, like all naturalness criteria, this one is inherently fuzzy, as $\delta$ could be significantly less than 1 , for example, because the corresponding coupling(s) happen to have a small magnitude and/or a phase alignment with the bare $\theta$. We will therefore not commit to a specific requirement for $p$, with the understanding that larger $p$ is safer in some sense.

Previous works exploring solutions to the axion quality problem have invoked composite axion models [35-45], additional continuous gauge symmetries [32,34,46-53], and discrete gauged symmetries in nonsupersymmetric [54-58] and supersymmetric [59-66] models. Here, we will be interested in the latter type of idea, in which the $U(1)$ PQ symmetry arises as an approximate accidental consequence of a discrete symmetry imposed on superfields. We will consider models in which discrete symmetries forbid PQ-violating Lagrangian terms up to some mass dimension and refer to the smallest exponent allowed in a particular model for the PQ-violating terms of the types in Eqs. (1.11) and (1.12) as the PQ-violation suppression $p$. Note that if the suppression $p$ for a particular model is odd, then it can be bumped up to the next integer by simply imposing another $Z_{2}$ symmetry under which both $X$ and $Y$ are odd, since this forbids all terms of the forms Eqs. (1.11) and (1.12) with odd $p$. We therefore consider as potentially 
viable any models whose discrete symmetries predict that $p$ should be 7 or more and consider models with $p \geq 12$ for all PQ-violating terms involving only $X$ and $Y$ as presumptively high quality.

Since the Kim-Nilles mechanism provides a $\mu$ term for the $H_{u}$ and $H_{d}$ fields at the TeV scale, it is reasonable to suppose that the same mechanism can give masses to other vectorlike pairs of chiral superfields as well, some of which could, therefore, be at the TeV scale just like the MSSM Higgs and Higgsino particles. For each additional pair $\Phi+\bar{\Phi}$ of chiral superfields, supersymmetric mass terms near the $\mathrm{TeV}$ scale can arise in three possible ways, due to nonrenormalizable superpotential terms of the form

$$
W_{\text {mass }}=\left\{\begin{array}{l}
\frac{\lambda_{\Phi}}{M_{P}} X Y \Phi \bar{\Phi} \\
\frac{\lambda_{\Phi}}{2 M_{P}} X^{2} \Phi \bar{\Phi} \\
\frac{\lambda_{\Phi}}{2 M_{P}} Y^{2} \Phi \bar{\Phi}
\end{array}\right.
$$

assuming $\lambda_{\Phi}$ is of the order of one. Alternatively, masses at the intermediate scale can be achieved by renormalizable superpotential terms of the form

$$
W_{\text {mass }}=\left\{\begin{array}{l}
\lambda_{\Phi} X \Phi \bar{\Phi} \\
\lambda_{\Phi} Y \Phi \bar{\Phi}
\end{array}\right.
$$

The net PQ charges of $\Phi \bar{\Phi}$ are important for understanding the low-energy axion couplings, as discussed in the next section. Table II shows the possible values of PQ charges $Q_{\Phi \bar{\Phi}} \equiv\left(Q_{\Phi}+Q_{\bar{\Phi}}\right)$ in the extensions of the four base models, for the various possible superpotential mass terms.

We will consider pairs of additional vectorlike quark or lepton superfields $\Phi+\bar{\Phi}$ chosen from among those in Table III. The first five pairs each include one field with the same color and electroweak quantum numbers as the MSSM chiral superfields, from which they are distinguished by the use of capital letters. While any of the possible mass terms could be used independently for each of types of fields, it is well known that nearly degenerate

TABLE II. Possible values of net PQ charges $Q_{\Phi \bar{\Phi}} \equiv\left(Q_{\Phi}+\right.$ $\left.Q_{\bar{\Phi}}\right)$ for vectorlike chiral superfield pairs, in the extensions of the four base models, depending on the possible superpotential terms that provide masses due to the scalar components of $X$ and $Y$ obtaining VEVs of the order of $M_{\text {int }}$. The first three mass terms provide for a $\mathrm{TeV}$-scale mass, and the last two provide for an intermediate-scale mass.

\begin{tabular}{lrrrr}
\hline \hline Mass terms & $\mathrm{B}_{\mathrm{I}}$ & $\mathrm{B}_{\mathrm{II}}$ & $\mathrm{B}_{\mathrm{III}}$ & $\mathrm{B}_{\text {IV }}$ \\
\hline$X Y \Phi \bar{\Phi}$ & -2 & 2 & $-2 / 3$ & 0 \\
$X^{2} \Phi \bar{\Phi}$ & 2 & -2 & $2 / 3$ & -2 \\
$Y^{2} \Phi \bar{\Phi}$ & -6 & 6 & -2 & 2 \\
$X \Phi \bar{\Phi}$ & 1 & -1 & $1 / 3$ & -1 \\
$Y \Phi \bar{\Phi}$ & -3 & 3 & -1 & 1 \\
\hline \hline
\end{tabular}

TABLE III. Vectorlike pairs of chiral superfields $\Phi+\bar{\Phi}$ that can be added to the base models and their Standard Model gauge transformation properties. These pairs will carry nonzero net PQ charges as shown in Table II, depending on the source of their mass.

\begin{tabular}{lc}
\hline \hline Superfields & $S U(3)_{c} \times S U(2)_{L} \times U(1)_{Y}$ \\
\hline$Q+\bar{Q}$ & $(\mathbf{3}, \mathbf{2}, 1 / 6)+(\overline{\mathbf{3}}, \mathbf{2},-1 / 6)$ \\
$U+\bar{U}$ & $(\mathbf{3}, \mathbf{1}, 2 / 3)+(\overline{\mathbf{3}}, \mathbf{1},-2 / 3)$ \\
$E+\bar{E}$ & $(\mathbf{1}, \mathbf{1},-1)+(\mathbf{1}, \mathbf{1}, 1)$ \\
$D+\bar{D}$ & $(\mathbf{3}, \mathbf{1},-1 / 3)+(\overline{\mathbf{3}}, \mathbf{1}, 1 / 3)$ \\
$L+\bar{L}$ & $(\mathbf{1}, \mathbf{2},-1 / 2)+(\mathbf{1}, \mathbf{2}, 1 / 2)$ \\
$D_{6}+\bar{D}_{6}$ & $(\mathbf{6}, \mathbf{1}, 1 / 3)+(\overline{\mathbf{6}}, \mathbf{1},-1 / 3)$ \\
\hline \hline
\end{tabular}

sets of chiral superfields in $\mathbf{5}+\overline{\mathbf{5}}=D+\bar{D}+L+\bar{L}$ or $\mathbf{1 0}+\overline{\mathbf{1 0}}=Q+\bar{Q}+U+\bar{U}+E+\bar{E}$ representations of the $S U(5)$ grand unified theory [67] will preserve the apparent unification of gauge couplings observed in the MSSM, as illustrated, for example, in the left panel in Fig. 1. Note that we do not assume that $S U(5)$ is actually the unbroken gauge group in the ultraviolet, and we allow for different components of the $\mathbf{5}+\overline{\mathbf{5}}$ and/or $\mathbf{1 0}+\overline{\mathbf{1 0}}$ to have different mass source terms and, therefore, different PQ charges. In Table III, we have also included a more exotic pair, an $S U(3)_{c}$ color sextet (quix) and its conjugate with electric charges $\pm 1 / 3$, denoted $D_{6}+\bar{D}_{6}$. If these have masses at an intermediate scale $M_{\text {int }} \approx 10^{11} \mathrm{GeV}$ and there are two $L+\bar{L}$ pairs near the TeV scale, then one can again have gauge coupling unification at a scale somewhat higher than in the MSSM, as shown in the right panel in Fig. 1. Although this combination might seem somewhat of an ad hoc choice, it is of interest, because it provides an example of an even more enhanced coupling of the axion to photons, as we will see.

There are other classes of quixotic models that can have gauge coupling unification, for example, $D_{6}+\bar{D}_{6}$ or $U_{6}+$ $\bar{U}_{6}=(\mathbf{6}, \mathbf{1},-2 / 3)+(\overline{\mathbf{6}}, \mathbf{1}, 2 / 3)$ quixes at the intermediate scale that have one or two weak isotriplets $T \sim(\mathbf{1}, \mathbf{3}, 0)$ at the same scale. Such models do not predict any new particles at the $\mathrm{TeV}$ scale and, therefore, are omitted from our discussion.

The rest of this paper is organized as follows. In Sec. II, we review the general properties of axion models, in particular, the relation between PQ charge assignments and axion couplings, the domain wall problem, and the axion quality problem. The relevant relations are obtained for supersymmetric DFSZ axion models of the type described above. In Sec. III, we explore possible discrete symmetries that can solve the axion quality problem and establish that there are many available for all of the base models and their extensions. In Sec. IV, we discuss axion signals and detection prospects for our models, including both present constraints and future detection prospects. Section V contains some summarizing remarks. 

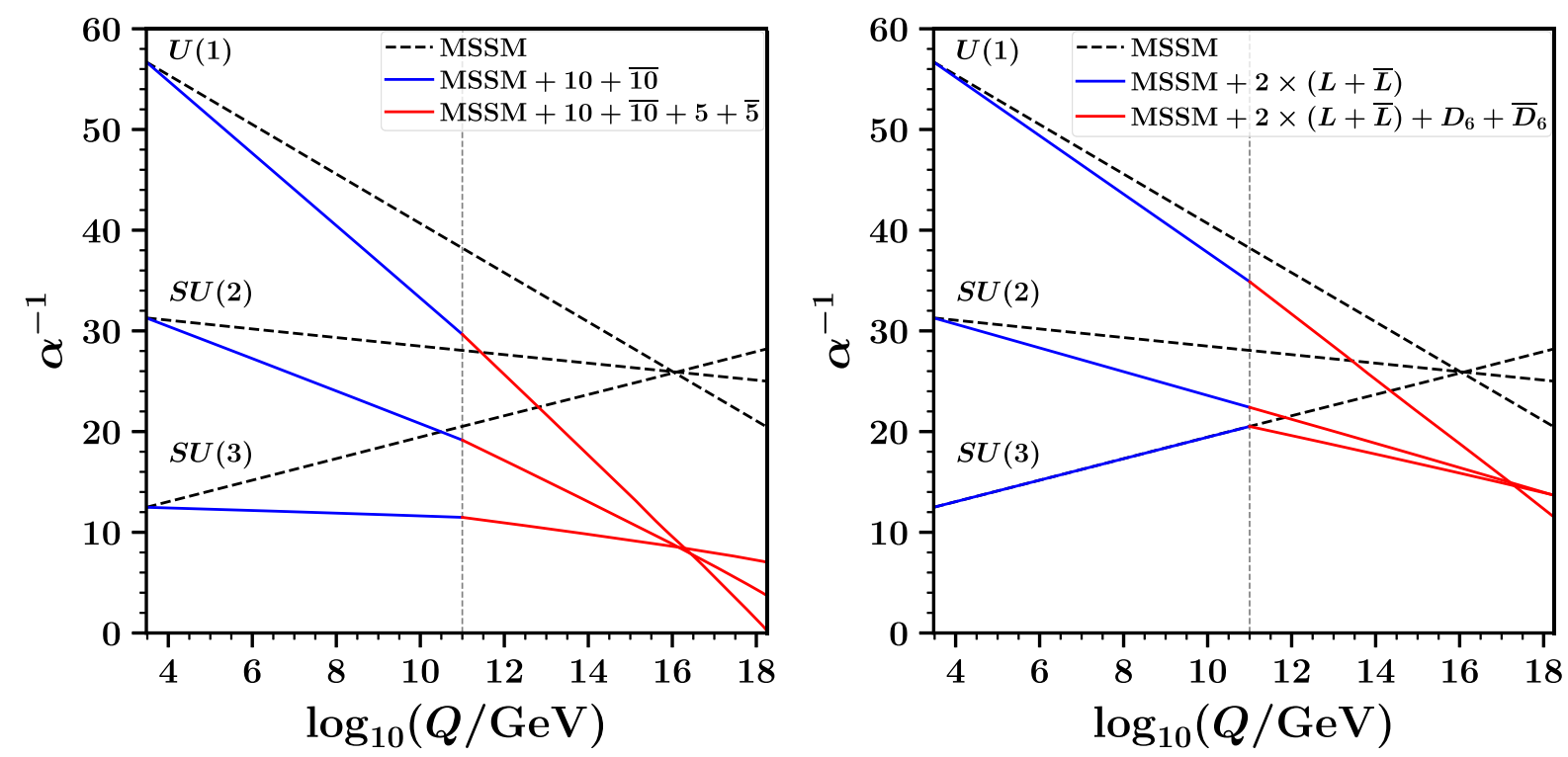

FIG. 1. Examples of gauge coupling unification with extra chiral supermultiplets added to the MSSM at both the TeV and $M_{\text {int }}$ scales. The left panel shows a typical example with extra vectorlike chiral supermultiplets in complete $S U(5)$ representations, in this case a $\mathbf{1 0}+\overline{\mathbf{1 0}}$ near the $\mathrm{TeV}$ scale and a $\mathbf{5}+\overline{\mathbf{5}}$ at the intermediate scale. The right panel shows a more exotic example with two pairs of vectorlike doublet leptons at the $\mathrm{TeV}$ scale and a pair of quixes at the intermediate scale $M_{\text {int }}=10^{11} \mathrm{GeV}$, leading to unification of couplings above $10^{17} \mathrm{GeV}$.

\section{PECCEI-QUINN CHARGES, ANOMALIES, AND AXION COUPLINGS}

\section{A. Axion properties in general}

In this section, we review the general properties of QCD axion models, following the discussion in Ref. [20]. The anomalous divergence of the Peccei-Quinn current in a model with each left-handed Weyl fermion $\psi_{f}$ transforming under $S U(3)_{c}$ in the representation $R_{f}$ with a PQ charge $Q_{f}$ (such that $\psi_{f} \rightarrow e^{i Q_{f} \alpha} \psi_{f}$ ) is given by

$$
\partial_{\mu} j_{\mathrm{PQ}}^{\mu}=\frac{g_{s}^{2}}{16 \pi^{2}} N G_{\mu \nu}^{a} \tilde{G}^{a \mu \nu}+\frac{e^{2}}{16 \pi^{2}} E F_{\mu \nu} \tilde{F}^{\mu \nu},
$$

where the $U(1)_{\mathrm{PQ}}-\left[S U(3)_{c}\right]^{2}$ and $U(1)_{\mathrm{PQ}}-\left[U(1)_{\mathrm{EM}}\right]^{2}$ anomaly coefficients, which play an important role in the low-energy phenomenology, are

$$
\begin{gathered}
N=\operatorname{Tr}\left[Q_{f} T\left(R_{f}\right)\right], \\
E=\operatorname{Tr}\left[Q_{f} q_{f}^{2}\right],
\end{gathered}
$$

respectively. Here, the trace in $N$ is over all left-handed fermion representations with $S U(3)_{c}$ index $T\left(R_{f}\right)$, while the trace in $E$ is over all left-handed fermion fields with electromagnetic (EM) charge $q_{f}$. The PQ current can be explicitly written as

$$
j_{\mathrm{PQ}}^{\mu}=-i \sum_{s} Q_{s}\left(\varphi_{s}^{\dagger} \partial^{\mu} \varphi_{s}-\varphi_{s} \partial^{\mu} \varphi_{s}^{\dagger}\right)-\sum_{f} Q_{f} \psi_{f}^{\dagger} \bar{\sigma}^{\mu} \psi_{f},
$$

where $\varphi_{s}$ are complex scalar fields, each with a PQ charge $Q_{s}$ (such that $\varphi_{s} \rightarrow e^{i Q_{s} \alpha} \varphi_{s}$ ).

The PQ symmetry can be spontaneously broken by nonzero VEVs of some scalars $\left\langle\varphi_{s}\right\rangle \equiv v_{s}$ (without breaking color or EM) that can be written as

$$
\varphi_{s}=\frac{1}{\sqrt{2}}\left(v_{s}+\rho_{s}\right) e^{i a_{s} / v_{s}}
$$

Here, $\rho_{s}$ and $a_{s}$ are the canonically normalized radial and angular fields, respectively. In our case, the scalars with VEVs will be either doublets or singlets under $S U(2)_{L}$. The PQ current becomes

$$
j_{\mathrm{PQ}}^{\mu}=\sum_{s} Q_{s} v_{s} \partial^{\mu} a_{s}-\sum_{f} Q_{f} \psi_{f}^{\dagger} \bar{\sigma}^{\mu} \psi_{f}
$$

in the decoupling limit, i.e., ignoring the terms with heavy radial fields $\rho_{s}$, which are PQ invariant. Now, defining

$$
v_{A}^{2}=\sum_{s} Q_{s}^{2} v_{s}^{2},
$$

the pseudo-Nambu-Goldstone boson axion field $A$ is given by

$$
A=\frac{1}{v_{A}} \sum_{s} Q_{s} v_{s} a_{s}
$$

This results in 


$$
j_{\mathrm{PQ}}^{\mu}=v_{A} \partial^{\mu} A-\sum_{f} Q_{f} \psi_{f}^{\dagger} \bar{\sigma}^{\mu} \psi_{f}
$$

We also note that, under a $U(1)_{\mathrm{PQ}}$ transformation parameterized by $\alpha$,

$$
\begin{gathered}
a_{s} \rightarrow a_{s}+\alpha Q_{s} v_{s}, \\
A \rightarrow A+\alpha v_{A} .
\end{gathered}
$$

Assuming the PQ symmetry is not explicitly broken, the PQ charges in a theory are determined by the terms in the Lagrangian. However, there is an ambiguity in the charges due to the freedom to add multiples of the weak hypercharge and any other nonanomalous $U(1)$ symmetries that might be present in the theory. The "physical" PQ charges can be obtained by imposing an orthogonality condition:

$$
\sum_{s} Y_{s} Q_{s} v_{s}^{2}=0
$$

to ensure that the axion and the $Z$ boson do not mix. Here, $Y_{s}$ are the $U(1)_{Y}$ weak hypercharges of the scalars that get VEVs. The above condition can be obtained by requiring the axion $A$ to be invariant under $U(1)_{Y}$ gauge transformations parameterized by $\alpha_{Y}$ :

$$
\begin{gathered}
a_{s} \rightarrow a_{s}+\alpha_{Y} Y_{s} v_{s}, \\
A \rightarrow A+\frac{\alpha_{Y}}{v_{A}} \sum_{s} Y_{s} Q_{s} v_{s}^{2}=A .
\end{gathered}
$$

The would-be Nambu-Goldstone boson $G$ that becomes the longitudinal component of the $Z$ boson is

$$
G=\frac{1}{v^{\prime}} \sum_{s} Y_{s} v_{s} a_{s}, \quad v^{2}=\sum_{s} Y_{s}^{2} v_{s}^{2} .
$$

The anomalous divergence of the PQ current in Eq. (2.1), with $j_{\mathrm{PQ}}^{\mu}$ in Eq. (2.9), now implies

$$
\begin{aligned}
\partial_{\mu} \partial^{\mu} A-\frac{1}{v_{A}} \sum_{f} Q_{f} \partial_{\mu}\left(\psi_{f}^{\dagger} \bar{\sigma}^{\mu} \psi_{f}\right) \\
=\frac{N}{v_{A}} \frac{g_{s}^{2}}{16 \pi^{2}} G_{\mu \nu}^{a} \tilde{G}^{a \mu \nu}+\frac{E}{v_{A}} \frac{e^{2}}{16 \pi^{2}} F_{\mu \nu} \tilde{F}^{\mu \nu} .
\end{aligned}
$$

This equation can be obtained as the Euler-Lagrange equation of the following effective Lagrangian:

$$
\begin{aligned}
\mathcal{L}_{A} \supset & \frac{1}{2} \partial_{\mu} A \partial^{\mu} A+\frac{g_{s}^{2}}{32 \pi^{2}} \frac{A}{f_{A}} G_{\mu \nu}^{a} \tilde{G}^{a \mu \nu}+\frac{E}{N} \frac{e^{2}}{32 \pi^{2}} \frac{A}{f_{A}} F_{\mu \nu} \tilde{F}^{\mu \nu} \\
& -\frac{1}{N} \frac{\partial_{\mu} A}{2 f_{A}} \sum_{f} Q_{f} \psi_{f}^{\dagger} \bar{\sigma}^{\mu} \psi_{f},
\end{aligned}
$$

where, provided that the PQ symmetry has a nonzero QCD anomaly $N$, the axion decay constant is defined by

$$
f_{A} \equiv \frac{v_{A}}{2 N}
$$

As a convention, we choose to define $f_{A}$ as always positive. In some models, $N$ is negative, in which case $v_{A}$ is negative in our convention.

In terms of four-component Dirac fermions $\Psi_{f}$ with lefthanded and conjugate of right-handed components carrying PQ charges $Q_{f}$ and $Q_{\bar{f}}$, respectively,

$$
\begin{aligned}
\mathcal{L}_{A} \supset & \frac{1}{2} \partial_{\mu} A \partial^{\mu} A+\frac{g_{s}^{2}}{32 \pi^{2}} \frac{A}{f_{A}} G_{\mu \nu}^{a} \tilde{G}^{a \mu \nu}+c_{\gamma} \frac{e^{2}}{32 \pi^{2}} \frac{A}{f_{A}} F_{\mu \nu} \tilde{F}^{\mu \nu} \\
+ & \frac{\partial_{\mu} A}{2 f_{A}} \sum_{f} c_{f} \bar{\Psi}_{f} \gamma^{\mu} \gamma_{5} \Psi_{f}+\frac{\partial_{\mu} A}{2 f_{A}} \sum_{f} c_{f}^{\prime} \bar{\Psi}_{f} \gamma^{\mu} \Psi_{f}
\end{aligned}
$$

with

$$
\begin{gathered}
c_{\gamma}=\frac{E}{N}, \\
c_{f}=\frac{Q_{f}+Q_{\bar{f}}}{2 N}, \\
c_{f}^{\prime}=\frac{Q_{\bar{f}}-Q_{f}}{2 N} .
\end{gathered}
$$

One can now express the axion-fermion couplings in terms of axial currents only, eliminating the vector currents. This can be achieved by redefining Dirac fermions $\Psi_{f}$ by the following $U(1)$ vector transformation (as opposed to the axial transformation that contributes to the PQ anomaly):

$$
\Psi_{f} \rightarrow e^{i c_{f}^{\prime} A / 2 f_{A}} \Psi_{f}
$$

under which the kinetic terms of the fermions give an additional contribution that cancels the axion-fermion couplings involving the vector currents:

$$
\begin{aligned}
\sum_{f} i \Psi_{f} \gamma^{\mu} \partial_{\mu} \Psi_{f} \rightarrow & \sum_{f} i \Psi_{f} \gamma^{\mu} \partial_{\mu} \Psi_{f} \\
& -\frac{\partial_{\mu} A}{2 f_{A}} \sum_{f} c_{f}^{\prime} \bar{\Psi}_{f} \gamma^{\mu} \Psi_{f}
\end{aligned}
$$

After evaluating the coefficients of axion-photon and axion-fermion couplings given in Eqs. (2.20) and (2.21), respectively, the resulting low-energy axion interaction Lagrangian can be obtained as 


$$
\begin{aligned}
\mathcal{L}_{A}= & \frac{1}{2} \partial^{\mu} A \partial_{\mu} A-\frac{1}{2} m_{A}^{2} A^{2}+C_{A \gamma} \frac{e^{2}}{32 \pi^{2}} \frac{A}{f_{A}} F_{\mu \nu} \tilde{F}^{\mu \nu} \\
& +\frac{\partial_{\mu} A}{2 f_{A}} \sum_{f=p, n, e} C_{A f} \bar{\Psi}_{f} \gamma^{\mu} \gamma_{5} \Psi_{f},
\end{aligned}
$$

where the axion mass is given in terms of the axion decay constant $f_{A}$ by [68]

$$
m_{A}=5.691(51) \frac{10^{12} \mathrm{GeV}}{f_{A}} \mu \mathrm{eV},
$$

and [69]

$$
\begin{gathered}
C_{A \gamma}=c_{\gamma}-1.92(4), \\
C_{A p}=-0.47(3)+0.88(3) c_{u}-0.39(2) c_{d}-C_{A, \mathrm{sea}} \\
C_{A n}=-0.02(3)+0.88(3) c_{d}-0.39(2) c_{u}-C_{A, \mathrm{sea}} \\
C_{A, \mathrm{sea}}=0.038(5) c_{s}+0.012(5) c_{c}+0.009(2) c_{b} \\
+0.0035(4) c_{t}, \\
C_{A e}=c_{e}
\end{gathered}
$$

The tree-level couplings to fermions above do not include the effects of renormalization group running [70-75], which are usually small compared to experimental uncertainties. A notable exception is the coupling $C_{A e}$ when $c_{e}$ vanishes or is very small in hadronic axion models, which we will include as nonsupersymmetric benchmarks below. In those cases, we include the leading logarithmic contribution $[70,71]$

$$
\Delta C_{A e}=\frac{3 \alpha^{2}}{4 \pi^{2}}\left(c_{\gamma} \ln \frac{f_{A}}{m_{e}}-1.92(4) \ln \frac{\mathrm{GeV}}{m_{e}}\right) .
$$

Finally, the effective Lagrangian which provides the axion coupling to photons and on-shell electrons, protons, and neutrons can be written as

$$
\mathcal{L}_{A}^{\text {int }}=\frac{1}{4} g_{A \gamma} A F_{\mu \nu} \tilde{F}^{\mu \nu}-\sum_{f=p, n, e} i g_{A f} A \bar{\Psi}_{f} \gamma_{5} \Psi_{f},
$$

with the definitions

$$
g_{A \gamma}=\frac{\alpha}{2 \pi} \frac{C_{A \gamma}}{f_{A}}, \quad g_{A f}=m_{f} \frac{C_{A f}}{f_{A}} .
$$

Here we note that the contribution from the $C P$-violating terms where the axion derivatively couples to the vector fermion current in Eq. (2.19) vanishes after integrating by parts and using the Dirac equation [without having to redefine the fermion fields as done in Eq. (2.23)].
After the PQ symmetry breaking, a discrete subgroup $Z_{N_{\mathrm{DW}}}\left(=e^{2 k \pi i / N_{\mathrm{DW}}}, k=0,1, \ldots, N_{\mathrm{DW}}-1\right)$ is left unbroken. $N_{\mathrm{DW}}$ is the domain wall (DW) number that corresponds to the number of discrete sets of inequivalent degenerate minima of the axion potential [76]. With the above definition of PQ-QCD-QCD anomaly coefficient $N$, the DW number can be computed as [77]

$$
N_{\mathrm{DW}} \equiv \text { minimum integer }\left(2 N \sum_{s} \frac{n_{s} Q_{s} v_{s}^{2}}{v_{A}^{2}}\right)
$$

where $n_{s} \in \mathbb{Z}$. The domain wall number must be invariant under the rescalings of the PQ charges, as is reflected in the above formula.

Models with $N_{\text {DW }}>1$ may have nontrivial cosmological implications. In particular, formation of topological defects such as stable domain walls, due to degenerate vacua with different possible phases of the axion, can dominate the early Universe [76]. However, the cosmological domain wall problem may not arise if PQ symmetry is broken in the preinflationary era and is not restored after inflation ends, so that the observable Universe today consists of a single patch that initially had a common value of $\theta$. Another possibility [78] is that the apparent $Z_{N_{\text {DW }}}$ discrete symmetry relating different vacua is actually embedded within a continuous gauged symmetry that is spontaneously broken at high energies. The $N_{\mathrm{DW}}$ apparently distinct vacua are then actually the same, being connected by the continuous gauge symmetry. In this paper, we will concentrate instead on the possibility that safety is achieved by $N_{\mathrm{DW}}=1$, in which case any domain walls that form are bounded by strings on their edges and are highly unstable [79]. This can be achieved using a strategy similar to that in Ref. [80], by introducing heavy fermions charged under the PQ symmetry, in our case components of chiral supermultiplets that are vectorlike under the Standard Model gauge group.

\section{B. Application to DFSZ axions in supersymmetry}

In the following, we consider supersymmetric DFSZtype axion models with two PQ-breaking gauge-singlet fields $X$ and $Y$ as discussed in the introduction, consisting of the four base models $\mathrm{B}_{\mathrm{I}}, \mathrm{B}_{\mathrm{II}}, \mathrm{B}_{\mathrm{III}}$, and $\mathrm{B}_{\mathrm{IV}}$ summarized in Table I, with possible extensions by additional vectorlike superfields that maintain approximate gauge coupling unification. The additional chiral superfield content in the models considered below are listed in Table III, with mass terms as in Table II.

From our choice of normalization in Table I, we get the following condition on the PQ charges of the MSSM Higgses:

$$
Q_{H_{u}}+Q_{H_{d}}=-2
$$


Requiring that the Yukawa terms in the MSSM superpotential are $U(1)_{\mathrm{PQ}}$ invariant, we can express the following PQ charge combinations-the only combinations that enter the low-energy axion-fermion couplings-in terms of $Q_{H_{u}}$ :

$$
\begin{gathered}
Q_{\bar{u}}+Q_{q}=-Q_{H_{u}}, \\
Q_{\bar{d}}+Q_{q}=2+Q_{H_{u}}, \\
Q_{\bar{e}}+Q_{\ell}=2+Q_{H_{u}} .
\end{gathered}
$$

We have used Eq. (2.36) in the last two equations. Using the above constraints, the PQ charges of all the MSSM fields can be expressed in terms of only three numbers $Q_{H_{u}}$, $Q_{q}$, and $Q_{\ell}$, which at this point are still unconstrained and, therefore, can be treated as free parameters. This corresponds to the freedom to add multiples of other $U(1)$ charges to the PQ symmetry charges.

In order to obtain the low-energy axion couplings and the DW number, the scalars (represented by the same symbols as their respective supermultiplets) that acquire a VEV are parameterized as

$$
\begin{array}{rlrl}
X & \supset \frac{v_{x}}{\sqrt{2}} e^{i a_{x} / v_{x}}, & & Y \supset \frac{v_{y}}{\sqrt{2}} e^{i a_{y} / v_{y}}, \\
H_{u}^{0} \supset \frac{v_{u}}{\sqrt{2}} e^{i a_{u} / v_{u}}, & & H_{d}^{0} \supset \frac{v_{d}}{\sqrt{2}} e^{i a_{d} / v_{d}},
\end{array}
$$

where $H_{u}^{0}$ and $H_{d}^{0}$ are the neutral MSSM Higgs scalars, $a_{s}=\left\{a_{x}, a_{y}, a_{u}, a_{d}\right\}$ are the pseudoscalar bosons that contribute to the axion, and $v_{x}, v_{y} \gg v_{u}, v_{d}$ in an invisible axion model.

Imposing the orthogonality condition Eq. (2.12) in each of the base models yields

$$
\frac{Q_{H_{d}}}{Q_{H_{u}}}=\frac{v_{u}^{2}}{v_{d}^{2}} \equiv \tan ^{2} \beta
$$

with $s_{\beta} \equiv \sin \beta=v_{u} / v, c_{\beta} \equiv \cos \beta=v_{d} / v$, and $v^{2}=v_{u}^{2}+v_{d}^{2}$. The orthogonality condition has essentially used the freedom to add arbitrary multiples of the $U(1)_{Y}$ charges to the fields. The PQ charges for the MSSM Higgs supermultiplets using Eq. (2.36), consistent with the Higgs VEVs and our normalization convention $Q_{H_{u}}+Q_{H_{d}}=-2$, are

$$
Q_{H_{u}}=-2 c_{\beta}^{2}, \quad Q_{H_{d}}=-2 s_{\beta}^{2},
$$

and we have, from Eq. (2.7),

$2 N f_{A}=v_{A}= \begin{cases}{\left[v_{x}^{2}+9 v_{y}^{2}+4 s_{\beta}^{2} c_{\beta}^{2} v^{2}\right]^{1 / 2}} & \left(\mathrm{~B}_{\mathrm{I}}, \mathrm{B}_{\mathrm{II}}\right), \\ {\left[\frac{1}{9} v_{x}^{2}+v_{y}^{2}+4 s_{\beta}^{2} c_{\beta}^{2} v^{2}\right]^{1 / 2}} & \left(\mathrm{~B}_{\mathrm{III}}\right) \\ {\left[v_{x}^{2}+v_{y}^{2}+4 s_{\beta}^{2} c_{\beta}^{2} v^{2}\right]^{1 / 2}} & \left(\mathrm{~B}_{\mathrm{IV}}\right)\end{cases}$

with $N$ to be given soon below. The contribution $4 s_{\beta}^{2} c_{\beta}^{2} v^{2}$ is numerically negligible.

Using Eqs. (2.37)-(2.39), the PQ charges of the rest of the MSSM chiral superfields can be fixed in terms of $Q_{q}$ and $Q_{\ell}$. We can also require that neutrino masses are provided by the superpotential version of the Weinberg operator $\left(H_{u} \ell\right)\left(H_{u} \ell\right)$. Equivalently, from the point of view of anomalies, we can introduce a gauge-singlet neutrino superfield $\bar{\nu}$ which has superpotential terms

$$
W_{\text {seesaw }}=\frac{1}{2} \lambda_{\bar{\nu}} S \bar{\nu} \bar{\nu}+y_{\bar{\nu}} H_{u} \ell \bar{\nu},
$$

where $S$ is a $S U(3)_{c} \times S U(2)_{L} \times U(1)_{Y}$ singlet, for example, either a bare mass term or $X$ or $Y$. This then gives us the further constraint

$$
Q_{\bar{\nu}}=-Q_{S} / 2, \quad Q_{\ell}=-Q_{H_{u}}+Q_{S} / 2,
$$

where $Q_{S}=0$ in the case of a bare seesaw mass $\left(\lambda_{\bar{\nu}} S \rightarrow M_{\bar{\nu}}\right)$.

The resulting PQ charge assignments for the MSSM chiral superfields are shown in Table IV. These physical PQ charge assignments also hold for any extensions of the base models, as long as there are no additional scalars with nonzero VEVs that can potentially feed into the orthogonality condition in Eq. (2.12). Also included for future

TABLE IV. The superfield charges of the Peccei-Quinn symmetry and two linearly independent anomaly-free $U(1)$ symmetries of the dimensionless part of the MSSM superpotential. Here, $\bar{\nu}$ represents either a gauge-singlet neutrino or the combination $-H_{u} \ell$ appearing in the nonrenormalizable Weinberg operator for neutrino masses. The physical Peccei-Quinn charges given here are obtained by imposing the orthogonality condition Eq. (2.12) and the normalization condition $Q_{H_{u}}+Q_{H_{d}}=-2$ and are given in terms of two free parameters $Q_{q}$ and $Q_{\ell}$ and the ratio of Higgs expectation values $\tan \beta=s_{\beta} / c_{\beta}$. If a seesaw neutrino mass term $S \bar{\nu} \bar{\nu}$ is included in the superpotential for some gauge-singlet field $S$ whose scalar component gets a VEV, then $2 T_{R}^{3}=2 Y-(B-L)$ is explicitly broken and $Q_{\ell}=2 c_{\beta}^{2}+Q_{S} / 2$ is fixed.

\begin{tabular}{lcccccccc}
\hline \hline & $H_{u}$ & $H_{d}$ & $q$ & $\ell$ & $\bar{u}$ & $\bar{d}$ & $\bar{e}$ & $\bar{\nu}$ \\
\hline $\mathrm{PQ}$ & $-2 c_{\beta}^{2}$ & $-2 s_{\beta}^{2}$ & $Q_{q}$ & $Q_{\ell}$ & $2 c_{\beta}^{2}-Q_{q}$ & $2 s_{\beta}^{2}-Q_{q}$ & $2 s_{\beta}^{2}-Q_{\ell}$ & $2 c_{\beta}^{2}-Q_{\ell}$ \\
$6 Y$ & 3 & -3 & 1 & -3 & -4 & 2 & 6 & 0 \\
$2 T_{R}^{3}$ & 1 & -1 & 0 & 0 & -1 & 1 & 1 & -1 \\
\hline \hline
\end{tabular}


reference are the charges for two nonanomalous familyindependent $U(1)$ symmetries; one is $6 Y$, the weak hypercharge normalized to integer charges for all fields, and the second is $2 T_{R}^{3}=2 Y-(B-L)$, also with integer charges.

Using Eqs. (2.2) and (2.3), the PQ anomaly coefficients in the base models and their extensions to include vectorlike fields are

$$
\begin{aligned}
N= & 3+\sum Q_{Q \bar{Q}}+\frac{1}{2} \sum Q_{U \bar{U}}+\frac{1}{2} \sum Q_{D \bar{D}} \\
& +\frac{5}{2} \sum Q_{D_{6} \bar{D}_{6}}, \\
E= & 6+\frac{5}{3} \sum Q_{Q \bar{Q}}+\frac{4}{3} \sum Q_{U \bar{U}}+\frac{1}{3} \sum Q_{D \bar{D}} \\
& +\sum Q_{L \bar{L}}+\sum Q_{E \bar{E}}+\frac{2}{3} \sum Q_{D_{6} \bar{D}_{6}},
\end{aligned}
$$

where the contributions $\sum Q_{\Phi \bar{\Phi}}$ can be read from Table II, depending on the origins of the $\Phi \bar{\Phi}$ mass terms. Note that, for all four base models, we have $N=3$ and $E=6$. The coefficients $c_{\gamma}$ and $c_{f}$ defined in Eqs. (2.20) and (2.21) in the base models and their extensions are then

$c_{\gamma}=\frac{E}{N}, \quad c_{u}=\frac{c_{\beta}^{2}}{N}, \quad c_{d}=\frac{s_{\beta}^{2}}{N}, \quad c_{e}=\frac{s_{\beta}^{2}}{N}$.

Note that all of $N, E, c_{\gamma}, c_{u}, c_{d}$, and $c_{e}$, and, therefore, the low-energy effective Lagrangian parameters Eqs. (2.27)(2.31) and (2.34), are independent of the choices of $Q_{q}$ and $Q_{\ell}$, and we arrive at

$$
\begin{gathered}
g_{A \gamma}=\frac{\alpha}{2 \pi f_{A}}(E / N-1.92(4)), \\
g_{A e}=\frac{m_{e}}{f_{A}} \frac{s_{\beta}^{2}}{N} \\
g_{A n}=\frac{m_{n}}{f_{A}}\left(-0.02(3)+\left[0.833(30)-1.239(37) c_{\beta}^{2}\right] / N\right),
\end{gathered}
$$

$g_{A p}=\frac{m_{p}}{f_{A}}\left(-0.47(3)+\left[-0.437(21)+1.302(37) c_{\beta}^{2}\right] / N\right)$

for the central value with parenthetical uncertainty estimates in our base models and extensions, parameterized only by $N$ and $E$. Note that the model-independent ( $N$-independent) part of the neutron coupling $g_{A n}$ suffers from an accidental cancellation [69].

We now compute the domain wall number (i.e., the number of independent degenerate minima of the axion potential) for each of the four base models listed in Table I using the formula given in Eq. (2.35).

\section{Model $\mathbf{B}_{\mathbf{I}}$}

Using Eq. (2.35), we have

$\sum_{s} \frac{n_{s} Q_{s} v_{s}^{2}}{v_{A}^{2}}=\frac{-n_{x} v_{x}^{2}+3 n_{y} v_{y}^{2}-\left(n_{u}+n_{d}\right) 2 s_{\beta}^{2} c_{\beta}^{2} v^{2}}{v_{x}^{2}+9 v_{y}^{2}+4 s_{\beta}^{2} c_{\beta}^{2} v^{2}}$,

where $n_{x}, n_{y}, n_{u}$, and $n_{d}$ are integers accompanying the scalars $X, Y, H_{u}$, and $H_{d}$, respectively. In the above expression, our goal is to arrange each $n_{s}$ such that Eq. (2.53) is an integer. This is obtained provided that $n_{y}=-3 n_{x}$ and $n_{u}+n_{d}=2 n_{x}$. Then from Eqs. (2.35) and (2.53) we find

$$
N_{\text {DW }}=\text { minimum integer }\left|2 N n_{x}\right|,
$$

where $n_{x}$ is a nonzero integer. Therefore, since $N=3$ for the MSSM field content, the domain wall number of the base model $\mathrm{B}_{\mathrm{I}}$ is $N_{\mathrm{DW}}=6$.

\section{Model $\mathrm{B}_{\mathrm{II}}$}

Following a similar procedure as above, we have

$$
\sum_{s} \frac{n_{s} Q_{s} v_{s}^{2}}{v_{A}^{2}}=\frac{n_{x} v_{x}^{2}-3 n_{y} v_{y}^{2}-\left(n_{u}+n_{d}\right) 2 s_{\beta}^{2} c_{\beta}^{2} v^{2}}{v_{x}^{2}+9 v_{y}^{2}+4 s_{\beta}^{2} c_{\beta}^{2} v^{2}}
$$

which is again an integer if $n_{y}=-3 n_{x}$ but this time with $n_{u}+n_{d}=-2 n_{x}$. The domain wall number is therefore given by

$$
N_{\text {DW }}=\text { minimum integer }\left|2 N n_{x}\right|,
$$

where again $n_{x}$ is a nonzero integer. For the base model $\mathrm{B}_{\mathrm{II}}$ with $N=3$, this again amounts to $N_{\mathrm{DW}}=6$.

\section{Model $\mathrm{B}_{\text {III }}$}

Proceeding as above, we begin with

$$
\sum_{s} \frac{n_{s} Q_{s} v_{s}^{2}}{v_{A}^{2}}=\frac{-\frac{1}{3} n_{x} v_{x}^{2}+n_{y} v_{y}^{2}-\left(n_{u}+n_{d}\right) 2 s_{\beta}^{2} c_{\beta}^{2} v^{2}}{\frac{1}{9} v_{x}^{2}+v_{y}^{2}+4 s_{\beta}^{2} c_{\beta}^{2} v^{2}}
$$

This will be an integer if $n_{y}=-3 n_{x}$ and $n_{u}+n_{d}=6 n_{x}$, resulting in

$$
N_{\mathrm{DW}}=\text { minimum integer }\left|6 N n_{x}\right|,
$$

for $n_{x}$ a nonzero integer. For the base model $\mathrm{B}_{\text {III }}$ with $N=3$, we obtain $N_{\text {DW }}=18$. 


\section{Model $\mathrm{B}_{\mathrm{IV}}$}

To calculate the domain wall number for this model, we start with

$$
\sum_{s} \frac{n_{s} Q_{s} v_{s}^{2}}{v_{A}^{2}}=\frac{n_{x} v_{x}^{2}-n_{y} v_{y}^{2}-\left(n_{u}+n_{d}\right) 2 s_{\beta}^{2} c_{\beta}^{2} v^{2}}{v_{x}^{2}+v_{y}^{2}+4 s_{\beta}^{2} c_{\beta}^{2} v^{2}} .
$$

This will be an integer if $n_{y}=-n_{x}$ and $n_{u}+n_{d}=-2 n_{x}$, with the result

$$
N_{\text {DW }}=\text { minimum integer }\left|2 N n_{x}\right|,
$$

where again $n_{x}$ is a nonzero integer. This gives $N_{\mathrm{DW}}=6$ for the base model $\mathrm{B}_{\mathrm{IV}}$.

The formulas for $N_{\mathrm{DW}}$ in Eqs. (2.54), (2.56), (2.58), and (2.60) in terms of $N$ are also applicable to any extensions of the models $\mathrm{B}_{\mathrm{I}}, \mathrm{B}_{\mathrm{II}}, \mathrm{B}_{\mathrm{III}}$, and $\mathrm{B}_{\mathrm{IV}}$, respectively, provided that there are no additional scalars getting VEVs. In each case, the value of the anomaly $N$ should be computed by including contributions from the other strongly interacting chiral superfields present in the model extension, as in Eqs. (2.46). Then $N_{\mathrm{DW}}$ is the smallest integer $\left|2 N n_{x}\right|$ (for extensions of base models $\mathrm{B}_{\mathrm{I}}, \mathrm{B}_{\mathrm{II}}$, and $\mathrm{B}_{\mathrm{IV}}$ ) or the smallest integer $\left|6 N n_{x}\right|$ (for extensions of base model $\mathrm{B}_{\mathrm{III}}$ ), where $n_{x}$ is a nonzero integer.

Although $N_{\text {DW }} \neq 1$ in the base models and many of their extensions, it follows from the preceding that it is possible to achieve $N_{\mathrm{DW}}=1$ models and avoid the cosmological domain wall problem in certain extensions. This requires

$$
\text { for } \begin{aligned}
N_{\mathrm{DW}} & =1 \\
N & = \begin{cases} \pm \frac{1}{2} & \text { in model extensions of } \mathrm{B}_{\mathrm{I}}, \mathrm{B}_{\mathrm{II}}, \text { and } \mathrm{B}_{\mathrm{IV}}, \\
\pm \frac{1}{6} & \text { in model extensions of } \mathrm{B}_{\mathrm{III}} .\end{cases}
\end{aligned}
$$

This occurs in a variety of extensions consistent with gauge coupling unification, all of which must have the property that the total index of $S U(3)_{c}$ chiral supermultiplets (i.e., the equivalent number of quark + antiquark supermultiplets) with mass at the intermediate scale $M_{\text {int }} \sim f_{A}$ is odd. However, this is, of course, a necessary but not sufficient condition. In the particular case that there is only a $\mathbf{5}+\overline{\mathbf{5}}$ at $M_{\text {int }}$ and no other additional vectorlike quarks, then from Eqs. (2.46) and (2.61) it follows that $N_{\mathrm{DW}}=1$ cannot be constructed with any of the possible $Q_{D \bar{D}}$ shown in Table II. Some of the model extensions with $N_{\text {DW }}=1$ are listed in Table V. They include a model with a quix-antiquix pair at an intermediate scale near $M_{\text {int }}=10^{11} \mathrm{GeV}$.

It should also be noted that the new heavy vectorlike particles in the extended models must be allowed to decay to Standard Model particles in order to avoid dangerous cosmological charged relics. This is easy to arrange, as the decays can be mediated by couplings to the Standard
Model quark and lepton superfields via Yukawa terms in the superpotential, in several different ways. For example, in models with an extra $\mathbf{5}+\overline{\mathbf{5}}$, the vectorlike weak isosinglet down-type quark can decay through any of the dimensionless couplings $H_{d} q \bar{D}$ or $\ell q \bar{D}$ or $\bar{u} \bar{d} \bar{D}$, while the vectorlike weak isodoublet lepton can decay through any of the superpotential couplings $H_{d} \bar{e} L$ or $\ell \bar{e} L$ or $q \bar{d} L$. It is easy to construct similar couplings that allow $Q+\bar{Q}, U+\bar{U}$, and $E+\bar{E}$ to decay in models that have an extra $\mathbf{1 0}+\overline{\mathbf{1 0}}$. In each case, in order to not overconstrain the system of equations determining the PQ charges, or the discrete symmetry charges discussed in the next section, one may select only one of the couplings for a given $\Phi+\bar{\Phi}$ pair. (This also avoids tree-level violation of baryon number and/or lepton number, which could lead to proton decay.) In models with quixes (the last two rows of Table V), they can decay via a superpotential term of the form $D_{6} \bar{u} \bar{d}$. This fixes the PQ charge to be $Q_{D_{6}}=2 Q_{q}-2$. The important point for the low-energy axion phenomenology is that the existence of all such Yukawa couplings maintains the freedom to choose the net PQ charges $Q_{\Phi \bar{\Phi}}$ consistently with the vectorlike mass terms and, therefore, does not affect the PQ anomalies.

\section{DISCRETE SYMMETRIES TO PROTECT $U(1)_{\mathrm{PQ}}$}

\section{A. Conventions and assumptions for discrete symmetries}

In this section, we consider discrete symmetries that can protect the Peccei-Quinn $U(1)$ symmetry up to dimension $p$ in the $X$ and $Y$ superfields in the superpotential. In equivalent language, the Peccei-Quinn symmetry is an accidental consequence of imposing the discrete symmetry. We consider as possibilities an Abelian discrete symmetry $Z_{n}$ or a discrete $R$ symmetry $Z_{n}^{R}$. In both cases, each chiral superfield $\Phi$ has integer charge $z_{\Phi}=0,1, \ldots n-1(\bmod n)$. It will be convenient to treat both cases in a unified framework. We therefore take the gauginos and the anticommuting coordinates $\theta_{\alpha}$ to have charge $r$, and each superpotential term is required to have total $Z_{n}^{R}$ charge $2 r$ $(\bmod n)$. For the case of ordinary $($ non- $R$ ) discrete symmetries, we take $r=0$. In both cases, all Lagrangian terms have total discrete charge 0 . [For continuous $R$ symmetries it is customary to take $r=1$ by a choice of normalization, but for discrete $R$ symmetries this is not always possible, since we require that all $Z_{n}^{R}$ charges for all fields are integers $(\bmod n)$.]

In Table VI, we list the allowed ${ }^{3}$ values of $n$ that can provide for a maximum possible suppression $p$, for the base models and their extensions. This means that the lowest

\footnotetext{
${ }^{3}$ Note that $n$ must be even for non- $R(r=0)$ symmetries $Z_{n}$ for model $\mathrm{B}_{\mathrm{IV}}$ and its extensions, in order to allow the term $X^{2} Y^{2}$ but forbid the term $X Y$.
} 
TABLE V. Models with domain wall number $N_{\mathrm{DW}}=1$, obtained by extending the base models to include vectorlike superfield combinations consistent with gauge coupling unification. Here $\mathbf{5}$ and $\mathbf{1 0}$ refer to chiral superfields with the same content as the corresponding $S U(5)$ representations. Note that at least one vectorlike pair at the intermediate scale is required. Only the mass terms of the strongly interacting fields, which affect the PQ-QCD-QCD anomaly $N$ and, therefore, $N_{\text {Dw }}$, are shown explicitly. Different combinations of mass terms for the $L, \bar{L}$ and/or $E, \bar{E}$ fields give rise to the possible $3 E / N$ values shown. For brevity, some models in the category with $\mathbf{1 0}+\overline{\mathbf{1 0}}$ pairs at both the TeV and $M_{\text {int }}$ scales are omitted when they have the same anomaly coefficients as ones shown. The cases in the last two lines include quixes at the intermediate scale, which can provide large values of $E / N$.

\begin{tabular}{|c|c|c|c|c|}
\hline Model extension & Base & Mass terms & $1 / N$ & $3 E / N$ \\
\hline $\mathbf{1 0}+\overline{\mathbf{1 0}}$ at $M_{\mathrm{int}}$ & $\mathrm{B}_{\mathrm{I}}$ & $Y Q \bar{Q}+X U \bar{U}$ & 2 & $20,-4$ \\
\hline $\begin{array}{l}\mathbf{5}+\overline{\mathbf{5}} \text { at } \mathrm{TeV} \\
\mathbf{5}+\overline{\mathbf{5}} \text { at } M_{\mathrm{int}}\end{array}$ & $\mathrm{B}_{\mathrm{I}}$ & $\begin{array}{l}X Y D \bar{D}+Y D^{\prime} \bar{D}^{\prime} \\
Y^{2} D \bar{D}+X D^{\prime} \bar{D}^{\prime}\end{array}$ & $\begin{array}{l}2 \\
2\end{array}$ & $\begin{array}{l}44,20,-4,-28 \\
44,20,-4,-28\end{array}$ \\
\hline $\begin{array}{l}\mathbf{5}+\overline{\mathbf{5}} \text { at } \mathrm{TeV}, \\
\mathbf{1 0}+\overline{\mathbf{1 0}} \text { at } M_{\text {int }}\end{array}$ & $\begin{array}{r}\mathrm{B}_{\mathrm{I}} \\
\mathrm{B}_{\mathrm{II}} \\
\mathrm{B}_{\mathrm{IV}}\end{array}$ & $\begin{array}{l}Y Q \bar{Q}+Y U \bar{U}+X^{2} D \bar{D} \\
Y Q \bar{Q}+X U \bar{U}+X Y D \bar{D} \\
X Q \bar{Q}+Y U \bar{U}+Y^{2} D \bar{D} \\
X Q \bar{Q}+X U \bar{U}+X^{2} D \bar{D} \\
X Q \bar{Q}+X U \bar{U}+X^{2} D \bar{D}\end{array}$ & $\begin{array}{r}-2 \\
-2 \\
-2 \\
2 \\
2\end{array}$ & $\begin{array}{c}68,44,20,-4 \\
44,20,-4,-28 \\
44,20,-4,-28 \\
68,44,20,-4 \\
32,20,8,-4\end{array}$ \\
\hline $\begin{array}{l}\mathbf{1 0}+\overline{\mathbf{1 0}} \text { at } \mathrm{TeV}, \\
\mathbf{5}+\overline{\mathbf{5}} \text { at } M_{\mathrm{int}}\end{array}$ & $\begin{array}{l}\mathrm{B}_{\mathrm{II}} \\
\mathrm{B}_{\mathrm{III}} \\
\mathrm{B}_{\mathrm{IV}}\end{array}$ & $\begin{array}{l}X Y Q \bar{Q}+X Y U \bar{U}+X D \bar{D} \\
X Y Q \bar{Q}+X^{2} U \bar{U}+Y D \bar{D} \\
X^{2} Q \bar{Q}+Y^{2} U \bar{U}+Y D \bar{D} \\
X^{2} Q \bar{Q}+X^{2} U \bar{U}+X D \bar{D} \\
Y^{2} Q \bar{Q}+X Y U \bar{U}+Y D \bar{D} \\
Y^{2} Q \bar{Q}+Y^{2} U \bar{U}+X D \bar{D} \\
X^{2} Q \bar{Q}+X Y U \bar{U}+X D \bar{D} \\
X^{2} Q \bar{Q}+X^{2} U \bar{U}+X D \bar{D} \\
X^{2} Q \bar{Q}+X^{2} U \bar{U}+Y D \bar{D}\end{array}$ & $\begin{array}{r}2 \\
2 \\
2 \\
-2 \\
6 \\
6 \\
2 \\
-2 \\
2\end{array}$ & $\begin{array}{c}20,-4,-28,-52 \\
44,20,-4,-28 \\
20,-4,-28,-52 \\
20,-4,-28,-52 \\
44,20,-4,-28 \\
20,-4,-28,-52 \\
32,20,8,-4 \\
20,8,-4,-16 \\
20,8,-4,-16\end{array}$ \\
\hline $\begin{array}{l}\mathbf{1 0}+\overline{\mathbf{1 0}} \text { at } \mathrm{TeV}, \\
\mathbf{1 0}+\overline{\mathbf{1 0}} \text { at } M_{\mathrm{int}}\end{array}$ & $\begin{array}{r}\mathrm{B}_{\mathrm{I}} \\
\mathrm{B}_{\mathrm{II}} \\
\mathrm{B}_{\mathrm{III}} \\
\mathrm{B}_{\mathrm{IV}}\end{array}$ & $\begin{array}{l}X Y Q \bar{Q}+X Y U \bar{U}+X Q^{\prime} \bar{Q}^{\prime}+Y U^{\prime} \bar{U}^{\prime} \\
X Y Q \bar{Q}+X^{2} U \bar{U}+Y Q^{\prime} \bar{Q}^{\prime}+X U^{\prime} \bar{U}^{\prime} \\
X^{2} Q \bar{Q}+X Y U \bar{U}+X Q^{\prime} \bar{Q}^{\prime}+X U^{\prime} \bar{U}^{\prime} \\
X Y Q \bar{Q}+Y^{2} U \bar{U}+Y Q^{\prime} \bar{Q}^{\prime}+Y U^{\prime} \bar{U}^{\prime} \\
Y^{2} Q \bar{Q}+X Y U \bar{U}+Y Q^{\prime} \bar{Q}^{\prime}+X U^{\prime} \bar{U}^{\prime} \\
X Y Q \bar{Q}+X^{2} U \bar{U}+X Q^{\prime} \bar{Q}^{\prime}+X U^{\prime} \bar{U}^{\prime} \\
X^{2} Q \bar{Q}+X Y U \bar{U}+X Q^{\prime} \bar{Q}^{\prime}+X U^{\prime} \bar{U}^{\prime} \\
X^{2} Q \bar{Q}+X Y U \bar{U}+X Q^{\prime} \bar{Q}^{\prime}+Y U^{\prime} \bar{U}^{\prime}\end{array}$ & $\begin{array}{r}-2 \\
-2 \\
2 \\
-6 \\
-6 \\
2 \\
-2 \\
2 \\
2\end{array}$ & $\begin{array}{c}68,44,20,-4 \\
44,20,-4,-28 \\
68,44,20,-4 \\
68,44,20,-4 \\
44,20,-4,-28 \\
20,8,-4,-16 \\
20,8,-4,-16 \\
32,20,8,-4\end{array}$ \\
\hline $\begin{array}{l}2 \times(L+\bar{L}) \text { at } \mathrm{TeV} \\
D_{6}+\bar{D}_{6} \text { at } M_{\mathrm{int}}\end{array}$ & $\begin{array}{r}\mathrm{B}_{\mathrm{II}} \\
\mathrm{B}_{\mathrm{IV}}\end{array}$ & $\begin{array}{l}X D_{6} \bar{D}_{6} \\
X D_{6} \bar{D}_{6}\end{array}$ & $\begin{array}{l}2 \\
2\end{array}$ & $\begin{array}{c}104,80,56,32,8 \\
56,44,32,20,8\end{array}$ \\
\hline
\end{tabular}

TABLE VI. Allowed $n$, for discrete symmetries $Z_{n}$ and discrete $R$ symmetries $Z_{n}^{R}$, that can provide a maximum possible suppression up to $p$ for the base models and their extensions. The meaning of $p$ is that the lowest dimension PQ-violating superpotential term(s) allowed by the discrete symmetry and involving only $X$ and $Y$ are of the form $X^{j} Y^{p-j}$. No anomaly cancellation constraints are imposed, yet.

\begin{tabular}{lcccc}
\hline \hline Max suppression & $Z_{n}, \mathrm{~B}_{\mathrm{I}, \mathrm{II}, \mathrm{III}}$ & $Z_{n}, \mathrm{~B}_{\mathrm{IV}}$ & $Z_{n}^{R}, \mathrm{~B}_{\mathrm{I}, \mathrm{II}, \mathrm{III}}$ & $Z_{n}^{R}, \mathrm{~B}_{\mathrm{IV}}$ \\
\hline$p \leq 6$ & $n \leq 12$ or $14,15,18$ & $n \leq 10$ & $n \leq 12$ or 14 & $n \leq 11$ or 14 \\
$p=7$ & $13,17,21$ & 14 & 13,15 & 13 \\
$p=8$ & $16,20,24$ & 12 & $16,17,18,19,20,22$ & $12,16,17$ \\
$p=9$ & $19,23,27$ & 18 & 21,23 & $15,19,20,22$ \\
$p=10$ & $22,26,30$ & 16 & $24,25,26,27,28,30,36$ & $18,23,25,26$ \\
$p=11$ & $25,29,33$ & 22 & 29,31 & $21,28,29$ \\
$p=12$ & $28,32,36$ & 20 & $32,33,34,35,38,44$ & $24,31,32,34$ \\
$p=13$ & $31,35,39$ & 26 & 37,39 & $27,35,37,38$ \\
$p \geq 14$ & $34,37,38$, or $\geq 40$ & 24, or $\geq 28$ & $40,41,42,43$, or $\geq 45$ & $30,33,36$, or $\geq 39$ \\
\hline \hline
\end{tabular}


dimension superpotential term that is allowed by the discrete symmetry but violates the PQ symmetry has dimension $p$ and so has the form $X^{j} Y^{p-j} / M_{P}^{p-3}$. From these results, we can conclude that $n$ must be at least 13 (for models $\mathrm{B}_{\mathrm{I}, \mathrm{II}, \mathrm{III}}$ ) or 12 (for model $\mathrm{B}_{\mathrm{IV}}$ ) even if the axion decay constant $f_{A}$ is as low as $10^{9} \mathrm{GeV}$ so that we can have $p=7$. For higher required $p$, the smallest possible values of the order of $n$ of the discrete symmetry group can be read from the table. Of course, not every discrete symmetry at the order of $n$ will provide the suppression listed.

We now consider the conditions imposed by anomaly cancellation on the discrete symmetry group, which depend on the discrete symmetry charges of the superfields that are charged under the Standard Model gauge group. For simplicity, we assume that the MSSM quark and lepton chiral superfields have charges that are generation independent. Using the symbol $z_{\Phi}$ for the additive $Z_{n}^{R}$ charge of the chiral superfield $\Phi$, the $Z_{n}^{R} \times G \times G$ anomalies for $G=S U(3)_{c}, S U(2)_{L}$, and $U(1)_{Y}$ are, respectively,

$$
\begin{aligned}
A_{3}= & 6 r+n_{g}\left(2 z_{q}+z_{\bar{u}}+z_{\bar{d}}-4 r\right)+2 \Delta_{Q \bar{Q}}+\Delta_{U \bar{U}} \\
& +\Delta_{D \bar{D}}+5 \Delta_{D_{6} \bar{D}_{6}},
\end{aligned}
$$

$$
\begin{aligned}
A_{2}= & 4 r+n_{g}\left(3 z_{q}+z_{l}-4 r\right)+z_{H_{u}}+z_{H_{d}}-2 r \\
& +3 \Delta_{Q \bar{Q}}+\Delta_{L \bar{L}},
\end{aligned}
$$

$$
\begin{aligned}
A_{1}= & n_{g}\left(z_{q}+3 z_{l}+8 z_{\bar{u}}+2 z_{\bar{d}}+6 z_{\bar{e}}-20 r\right) \\
& +3\left(z_{H_{u}}+z_{H_{d}}-2 r\right) \\
& +\Delta_{Q \bar{Q}}+3 \Delta_{L \bar{L}}+8 \Delta_{U \bar{U}}+2 \Delta_{D \bar{D}} \\
& +6 \Delta_{E \bar{E}}+4 \Delta_{D_{6} \bar{D}_{6}} .
\end{aligned}
$$

Here, we have adopted a convenient but somewhat unusual normalization, by taking the index for the fundamental representation of the non-Abelian groups to be 1 rather than $1 / 2$, so that $A_{2}$ and $A_{3}$ are integers defined $(\bmod n)$. Likewise, the anomaly $A_{1}$ has been given in a normalization so that it is always an integer (assuming the weak hypercharge quantization of the Standard Model) and again defined $(\bmod n)$. The first term in each of $A_{3}$ and $A_{2}$ is the gaugino contribution. The number of chiral quark and lepton generations is $n_{g}=3$. Finally, the contributions $\Delta_{\Phi \bar{\Phi}}$ are equal to the sum of the fermion discrete symmetry charges $z_{\Phi}-r$ and $z_{\bar{\Phi}}-r$ for the indicated vectorlike chiral superfields and are also equal to the negative of the sum of $z_{X}$ and $z_{Y}$ contributions for the terms in Eqs. (1.18) and (1.19) that produce their masses.

With our normalization of the $A_{i}$, the conditions [61, 81-84] for the discrete symmetry to be anomaly-free can be obtained by treating $Z_{n}^{R}$ as a subgroup of an anomaly-free continuous $U(1)$ symmetry that is spontaneously broken by a scalar field VEV of charge $n$ [85], in the presence of other possible very heavy fermions. The anomaly-free condition is that one must have

$$
\frac{A_{3}+m_{3} n}{k_{3}}=\frac{A_{2}+m_{2} n}{k_{2}}=\frac{A_{1}+m_{1} n}{5 k_{1}}=\rho_{\mathrm{GS}},
$$

where $\rho_{\mathrm{GS}}$ is a constant that can arise from the GreenSchwarz (GS) mechanism [86] that may be used to cancel the anomalies for the $U(1)$ group that contains $Z_{n}^{R}, m_{1,2,3}$ are integers, and $k_{2,3}$ are positive integer Kac-Moody levels, while $k_{1}$, in general, could be arbitrary. For simplicity, we will therefore consider as a weak assumption that $k_{2}=k_{3}$ but also consider as a stronger assumption motivated by gauge coupling unification that $k_{3}=k_{2}=k_{1}=1$, corresponding to the normalization in which $S U(3)_{c} \times S U(2)_{L} \times U(1)_{Y}$ could be embedded in a simple grand unified theory group, $S U(5)$ or $S O(10)$ or $E_{6}$. We then have

$$
A_{2}=A_{3} \quad(\bmod n),
$$

for the weaker condition in which the $U(1)_{Y}$ normalization is considered arbitrary, with the additional stronger condition

$$
A_{1}=5 A_{3} \quad(\bmod n)
$$

if the gauge couplings are required to unify in the usual way. The value $\rho_{\mathrm{GS}}=0$ corresponds to the notable special case in which the Green-Schwarz mechanism does not play a role, in which case

$$
A_{1}=A_{2}=A_{3}=0 \quad(\bmod n) .
$$

From the requirement that the MSSM superpotential couplings are allowed, we have $z_{\bar{u}}=-z_{H_{u}}-z_{q}+2 r, z_{\bar{d}}=$ $-z_{H_{d}}-z_{q}+2 r$, and $z_{\bar{e}}=-z_{H_{d}}-z_{\ell}+2 r$. Furthermore, $z_{H_{d}}$ is determined either by $z_{H_{d}}=-z_{H_{u}}-z_{X}-z_{Y}+2 r$ for base model $\mathrm{B}_{\mathrm{I}}$ and its extensions, $z_{H_{d}}=-z_{H_{u}}-2 z_{X}+2 r$ for base models $\mathrm{B}_{\mathrm{II}}$ and $\mathrm{B}_{\mathrm{IV}}$ and their extensions, or $z_{H_{d}}=-z_{H_{u}}-2 z_{Y}+2 r$ for base model $\mathrm{B}_{\mathrm{III}}$ and its extensions. We also require that neutrino masses are provided by the superpotential version of the Weinberg operator $\left(H_{u} \ell\right)\left(H_{u} \ell\right)$. Equivalently, from the point of view of anomalies and low-energy phenomenology, we can introduce three gauge-singlet neutrino superfields $\bar{\nu}$ which have superpotential terms

$$
W_{\text {seesaw }}=\frac{1}{2} M_{\bar{\nu}} \bar{\nu} \bar{\nu}+y_{\bar{\nu}} H_{u} \ell \bar{\nu},
$$

and then we have that $z_{\bar{\nu}}=2 r-z_{\ell}-z_{H_{u}}$ must either be $r$ or perhaps $r+n / 2$ if $n$ is even.

If we temporarily neglect the $M_{\bar{\nu}}$ term, the superpotential $y_{u} H_{u} q \bar{u}-y_{d} H_{d} q \bar{d}-y_{e} H_{d} l \bar{e}+y_{\bar{\nu}} H_{u} \ell \bar{\nu}$ is invariant under two anomaly-free $U(1)$ symmetries with integer charges. 
One is $U(1)_{6 Y}$, where $Y$ is the weak hypercharge, and the other can be taken to be either $3(B-L)$ or $2 T_{R}^{3}=$ $2 Y-(B-L)$. The charges were listed in Table IV. It is apparent that we can always redefine the $Z_{n}^{R}$ charges by adding a multiple of $6 Y$ in order to make $q=0$, as a convention without loss of generality. Now, if $n$ is even, we can add $(n / 2) 2 T_{R}^{3}$ to every $z_{\Phi}$ to obtain another discrete symmetry which has the same anomalies $A_{1,2,3}$ and differs from the original discrete symmetry only by adding charges $n / 2(\bmod n)$ for the fields $H_{u}, H_{d}, \bar{u}, \bar{d}, \bar{e}$, and $\bar{\nu}$. This amounts to simply toggling whether matter-parity-violating terms are allowed or forbidden by $Z_{n}^{R}$. We therefore always take $z_{\bar{\nu}}=r$, rather than the alternative $z_{\bar{\nu}}=r+n / 2$ in the case that $n$ is even, with the understanding that there is always a corresponding discrete symmetry that can be obtained in this way. In the case of odd $n$, one can also always choose to impose matter parity or not.

All of the charges and $r$ are defined $(\bmod n)$. If $n$ is even, then any $Z_{n}^{R}$ symmetry with $r=n / 2$ is equivalent to the corresponding $Z_{n}$ non- $R$ symmetry from the low-energy point of view, because the two symmetries forbid and allow precisely the same Lagrangian terms. Also, if $r>n / 2$, then one can replace $\left(z_{\Phi}, r\right) \rightarrow\left(n-z_{\Phi}, n-r\right)$ to obtain an equivalent version of the discrete symmetry. Therefore, for the $R$ symmetries, one can take $0<r<n / 2$ without loss of generality.

For any given discrete symmetry, equivalent versions of it can be obtained by multiplying all of the charges (including $r$ ) by a common integer relatively prime to $n$ and then taking the results $(\bmod n)$. We use this to eliminate redundant low-energy descriptions of a given discrete symmetry. We also reduce every discrete symmetry to the smallest $n$ that describes it, by removing any common factors from the list of charges $z_{\Phi}$ and $r$.

It follows that the $Z_{n}$ or $Z_{n}^{R}$ charges of the base model chiral fields can always be written, by a conventional choice, in terms of only two independent charges $x$ and $h$ (and one binary choice $m=0,1$ for model $\mathrm{B}_{\mathrm{IV}}$ when $n$ is even and $r \neq 0$ ), as summarized in Table VII. Therefore, for the sake of brevity, we will not list discrete symmetry charges for $H_{d}$ and the MSSM quark and lepton fields below, since they can be obtained from Table VII. For extensions of the base models, the total discrete symmetry charges for each vectorlike pair can be obtained from those for $X$ and/or $Y$, given the mass term as specified in Eq. (1.18) for TeV-scale masses or Eq. (1.19) for intermediate-scale masses.

Gaugino masses, and holomorphic soft-supersymmetrybreaking terms corresponding to terms in the superpotential, are forbidden by unbroken discrete $R$ symmetries. Since they are a phenomenological necessity, a discrete $R$ symmetry must be spontaneously broken. This can be done with a spurion with $Z_{n}^{R}$ charge 0 , whose $F$-term component (with $Z_{n}^{R}$ charge equal to $-2 r$ ) obtains a VEV. In the lowenergy theory, this will give rise to the usual MSSM supersymmetry-breaking terms including gaugino masses, as well as PQ-violating terms of the form in Eq. (1.12). As already noted in the introduction, the resulting axion tadpole contribution is parametrically of the same order as the corresponding superpotential terms in Eq. (1.11) and, therefore, has the same order-of-magnitude effect on $\theta_{\text {eff }}$.

\section{B. Discrete non- $R$ symmetries $Z_{n}$}

In Table VIII, we give a complete list of the possible distinct non- $R(r=0) Z_{n}$ symmetries for the base models, consistent with a bare Weinberg operator $\left(H_{u} \ell\right)\left(H_{u} \ell\right)$ (or, equivalently, $\bar{\nu} \bar{\nu}$ ) for neutrino masses and the anomaly constraint $A_{2}=A_{3}(\bmod n)$. In the second column in the table, we show the resulting suppression factor $p$. We see that there is a large selection of $Z_{n}$ symmetries that satisfy the weaker anomaly-free constraint. These include, for example, a $Z_{22}$ symmetry with $p=11$ for base model $\mathrm{B}_{\mathrm{IV}}$, equivalent to one previously proposed and studied in Ref. [60]. In our Table VIII, this symmetry has $\left(X, Y, H_{u}\right)$ charges $(2,6 k+3,4 k-2)$ with $k=1$; the charges listed in Ref. [60] are different, but the symmetry is equivalent in the sense discussed above. There are many other discrete symmetries with equal or greater suppression $p$. For example, with the same base model $\mathrm{B}_{\mathrm{IV}}$, we see that there are also $Z_{16}$ and $Z_{20}$ symmetries that provide protection up to $p=10$ and 12 , respectively.

Despite this infinite number of possible $Z_{n}$ symmetries, the only base model cases that satisfy the additional condition $A_{1}=5 A_{3}(\bmod n)$ and have adequate suppression $(p \geq 7)$ of $\mathrm{PQ}$-violating terms in the superpotential are $Z_{36}$ for model $\mathrm{B}_{\text {III }}$ with $p=12$ (with three distinct possibilities

TABLE VII. Charges for discrete symmetry $\left(Z_{n}\right.$ for $r=0$, or $Z_{n}^{R}$ for nonzero integer $\left.0<r<n / 2\right)$, for chiral superfields in the base models, in terms of two integers $h$ and $x$, in the convention adopted here. In the case of $\mathrm{B}_{\mathrm{IV}}, m=0$ for odd $n$, while $m=0,1$ for even $n$ and $r \neq 0$, and $m=1$ for even $n$ if $r=0$. If vectorlike pairs are added to the model, their net discrete symmetry charges can be obtained from those for $X$ and/or $Y$, given the mass term as specified in Eq. (1.18) for TeV-scale masses or Eq. (1.19) for intermediate-scale masses.

\begin{tabular}{lcccccccccc}
\hline \hline & $X$ & $Y$ & $H_{u}$ & $H_{d}$ & $q$ & $\ell$ & $\bar{u}$ & $\bar{d}$ & $\bar{e}$ \\
\hline $\mathrm{B}_{\mathrm{I}}$ & $x$ & $2 r-3 x$ & $h$ & $2 x-h$ & 0 & $r-h$ & $2 r-h$ & $h-2 x+2 r$ & $2 h-2 x+r$ & $r$ \\
$\mathrm{~B}_{\mathrm{II}}$ & $x$ & $2 r-3 x$ & $h$ & $2 r-2 x-h$ & 0 & $r-h$ & $2 r-h$ & $h+2 x$ & $2 h+2 x-r$ & $r$ \\
$\mathrm{~B}_{\mathrm{III}}$ & $x$ & $2 r-3 x$ & $h$ & $6 x-h-2 r$ & 0 & $r-h$ & $2 r-h$ & $h-6 x+4 r$ & $2 h-6 x+3 r$ & $r$ \\
$\mathrm{~B}_{\mathrm{IV}}$ & $x$ & $r-x+m n / 2$ & $h$ & $2 r-2 x-h$ & 0 & $r-h$ & $2 r-h$ & $h+2 x$ & $2 h+2 x-r$ & $r$ \\
\hline \hline
\end{tabular}


TABLE VIII. Exhaustive list of distinct $Z_{n}$ symmetries that satisfy the discrete anomaly cancellation constraint $A_{2}=A_{3}(\bmod n)$ for the MSSM base models including the bare Weinberg operator $\left(H_{u} \ell\right)\left(H_{u} \ell\right)$ for neutrino masses. The meaning of $p$ is that the lowest dimension PQ-violating superpotential term(s) allowed by the discrete symmetry and involving only $X$ and $Y$ are of the form $X^{j} Y^{p-j}$. In this table, $k$ is any integer, and $m=0,1,2$. The middle three columns are the $Z_{n}$ charges for $X, Y$, and $H_{u}$, respectively. The $H_{d}$ and quark and lepton superfield charges are then determined as in Table VII, with the charges of $q$ and $H_{u} \ell$ set equal to 0 without loss of generality, as discussed in the text. Despite this infinite number of possible $Z_{n}$ symmetries, the only cases that satisfy the additional condition $A_{1}=5 A_{3}(\bmod n)$ and have adequate suppression of $\mathrm{PQ}$-violating terms $(p \geq 7)$ are $Z_{36}$ for model $\mathrm{B}_{\text {III }}$ with $p=k=12$ and $Z_{36}$ for model $\mathrm{B}_{\mathrm{IV}}$ with $p=8$ and $k=1$.

\begin{tabular}{|c|c|c|c|c|c|c|c|}
\hline Model & $n$ & $p$ & $X$ & $Y$ & $H_{u}$ & $A_{2}=A_{3}$ & $A_{1}$ \\
\hline \multirow[t]{5}{*}{$\mathrm{B}_{\mathrm{I}}$} & $3 k+1$ & $k+3$ & 1 & -3 & $k+3$ & -6 & -18 \\
\hline & $3 k+2$ & $k+2$ & 1 & -3 & $-k+2$ & -6 & -18 \\
\hline & $9 k$ & k & 3 & -9 & 8 & -18 & -54 \\
\hline & $9 k+3$ & $k+3$ & 3 & -9 & 8 & -18 & -54 \\
\hline & $9 k+6$ & $k+2$ & 3 & -9 & 8 & -18 & -54 \\
\hline \multirow[t]{5}{*}{$\mathrm{B}_{\mathrm{II}}$} & $3 k+1$ & $k+3$ & 1 & -3 & $-k-3$ & 6 & 18 \\
\hline & $3 k+2$ & $k+2$ & 1 & -3 & $k-2$ & 6 & 18 \\
\hline & $9 k$ & $k$ & 3 & -9 & $3 k-8$ & 18 & 54 \\
\hline & $9 k+3$ & $k+3$ & 3 & -9 & $3 k-7$ & 18 & 54 \\
\hline & $9 k+6$ & $k+2$ & 3 & -9 & $-3 k-10$ & 18 & 54 \\
\hline \multirow[t]{5}{*}{$\mathrm{B}_{\mathrm{III}}$} & $3 k$ & $k$ & 1 & -3 & $8+m k$ & -18 & -54 \\
\hline & $3 k+1$ & $k+3$ & 1 & -3 & 8 & -18 & -54 \\
\hline & $3 k+2$ & $k+2$ & 1 & -3 & 8 & -18 & -54 \\
\hline & $9 k+3$ & $k+3$ & 3 & -9 & $3 k+25$ & -54 & -162 \\
\hline & $9 k+6$ & $k+2$ & 3 & -9 & $3 k+26$ & -54 & -162 \\
\hline \multirow[t]{15}{*}{$\mathrm{B}_{\mathrm{IV}}$} & $12 k+2$ & $6 k+1$ & 1 & $6 k$ & $4 k-2$ & 6 & 18 \\
\hline & $12 k+2$ & $6 k+1$ & 2 & $6 k-1$ & $-4 k-6$ & 12 & 36 \\
\hline & $12 k+4$ & $6 k+4$ & 1 & $6 k+1$ & $-4 k-4$ & 6 & 18 \\
\hline & $12 k+8$ & $6 k+6$ & 1 & $6 k+3$ & $4 k$ & 6 & 18 \\
\hline & $12 k+10$ & $6 k+5$ & 1 & $6 k+4$ & $-4 k-6$ & 6 & 18 \\
\hline & $12 k+10$ & $6 k+5$ & 2 & $6 k+3$ & $4 k-2$ & 12 & 36 \\
\hline & $36 k$ & $6 k+2$ & 3 & $18 k-3$ & $12 k-8$ & 18 & 54 \\
\hline & $36 k+6$ & $6 k+1$ & 3 & $18 k$ & $-12 k-10$ & 18 & 54 \\
\hline & $36 k+6$ & $6 k+1$ & 6 & $18 k-3$ & $12 k-14$ & 36 & 108 \\
\hline & $36 k+12$ & $6 k+4$ & 3 & $18 k+3$ & $12 k-4$ & 18 & 54 \\
\hline & $36 k+18$ & $6 k+3$ & 3 & $18 k+6$ & $12 k-2$ & 18 & 54 \\
\hline & $36 k+18$ & $6 k+3$ & 6 & $18 k+3$ & $12 k-10$ & 36 & 108 \\
\hline & $36 k+24$ & $6 k+6$ & 3 & $18 k+9$ & $-12 k-16$ & 18 & 54 \\
\hline & $36 k+30$ & $6 k+5$ & 3 & $18 k+12$ & $12 k+2$ & 18 & 54 \\
\hline & $36 k+30$ & $6 k+5$ & 6 & $18 k+9$ & $-12 k-26$ & 36 & 108 \\
\hline
\end{tabular}

for the $H_{u}$ charge, labeled in the table by $\left.m=0,1,2\right)$ and $Z_{36}$ for model $\mathrm{B}_{\mathrm{IV}}$ with $p=8$. In both cases, the GreenSchwarz mechanism is needed, as $\rho_{\mathrm{GS}}=A_{3}=18(\bmod 36)$.

If there are extra vectorlike chiral supermultiplets that owe their masses to $X$ and $Y$, then there are so many possible discrete symmetries that we cannot provide an exhaustive list. Some examples are given in Tables IX and $\mathrm{X}$, in which we have limited ourselves to cases with at most one $\mathbf{5}+\overline{\mathbf{5}}$ or $\mathbf{1 0}+\overline{\mathbf{1 0}}$ of $S U(5)$ at the $\mathrm{TeV}$ scale and in which both anomaly-free constraints $A_{2}=A_{3}(\bmod n)$ and $A_{1}=5 A_{3}(\bmod n)$ are required. The order of $n$, the $Z_{n}$ MSSM charges, and the suppression $p$ for these symmetries are the same as found in Table VIII, but the anomalies $N$ and $E$ that enter into the low-energy axion phenomenology are different because of the contributions of the vectorlike fields. We also note that there are several examples that have $\rho_{\mathrm{GS}}=0$ and, therefore, do not require the Green-Schwarz mechanism. The examples with $\rho_{\mathrm{GS}}=0$ and $N \neq 0$ that have the smallest order of $n$ include $Z_{20}$ for extensions of $\mathrm{B}_{\mathrm{II}}$ and $\mathrm{B}_{\mathrm{III}}$ and $Z_{12}$ and $Z_{36}$ for extensions of $\mathrm{B}_{\mathrm{IV}}$. Each of these has $p=8$, which provides sufficient quality for the PQ symmetry for $f_{A} \lesssim 4 \times 10^{9} \mathrm{GeV}$ if the PQ-violating terms have order one couplings with generic phases and for larger $f_{A}$ otherwise. We note that, when $n$ is not too large, $\rho_{\mathrm{GS}} \neq 0$ tends to allow for higher $p$.

Also shown in Tables IX and $\mathrm{X}$ are some examples that have $\rho_{\mathrm{GS}}=0$ but also $N=0$, so that there is no PQ-QCDQCD anomaly. These examples will have a light axionlike particle but will not provide a solution to the strong $C P$ problem. One peculiar case is that of base model $\mathrm{B}_{\mathrm{IV}}$ extended by $X^{2} Q \bar{Q}+X^{2} U \bar{U}+X Y E \bar{E}$. This particular vectorlike content implies a completely anomaly-free PQ 
TABLE IX. Some examples of non- $R$ discrete symmetries $Z_{n}$ satisfying the anomaly cancellation conditions $A_{2}=A_{3}(\bmod n)$ and $A_{1}=5 A_{3}(\bmod n)$, obtained from base models $\mathrm{B}_{\mathrm{I}}, \mathrm{B}_{\mathrm{II}}$, or $\mathrm{B}_{\mathrm{III}}$ by adding up to one $\mathbf{5}+\overline{\mathbf{5}}$ or $\mathbf{1 0}+\overline{\mathbf{1 0}}$ of $S U(5)$ at the TeV scale. The suppression of PQ violation $p$ is defined so that the lowest dimension PQ-violating superpotential term(s) allowed by the discrete symmetry and involving only $X$ and $Y$ are of the form $X^{j} Y^{p-j}$. In all cases in this table, the $X$ and $Y$ charges are, respectively, 1 and -3 , and the $H_{u}$ charges are as listed with $m=0,1,2$. The $H_{d}$ and quark and lepton superfield charges are then determined as in Table VII. The last three columns give the Green-Schwarz contribution $\rho_{\mathrm{GS}}$ to the $Z_{n}$ anomalies and the PQ-QCD-QCD and PQ-EM-EM anomalies $N$ and $E$, respectively. The five cases with $\rho_{\mathrm{GS}}=0$ do not require a Green-Schwarz mechanism. However, of those, the two cases with $N=0$ have no PQ-QCD-QCD anomaly and, therefore, have an axionlike particle but do not provide a solution to the strong $C P$ problem.

\begin{tabular}{|c|c|c|c|c|c|c|c|}
\hline$\underline{\text { Base }}$ & Extension & $n$ & $p$ & $H_{u}$ & $\rho_{\mathrm{GS}}$ & $N$ & $3 E$ \\
\hline \multirow[t]{7}{*}{$\overline{\mathrm{B}_{\mathrm{I}}}$} & $X^{2} D \bar{D}+Y^{2} L \bar{L}$ & 20 & 8 & 12 & 12 & 4 & 2 \\
\hline & $Y^{2} D \bar{D}+X^{2} L \bar{L}$ & 36 & 12 & $12 m$ & 0 & 0 & 18 \\
\hline & $X^{2} D \bar{D}+X Y L \bar{L}$ & 36 & 12 & $4+12 m$ & 28 & 4 & 14 \\
\hline & $Y^{2} Q \bar{Q}+X Y U \bar{U}+X^{2} E \bar{E}$ & 36 & 12 & $4+12 m$ & 8 & -4 & -14 \\
\hline & $X^{2} Q \bar{Q}+Y^{2} U \bar{U}+Y^{2} E \bar{E}$ & 20 & 8 & 0 & 16 & 2 & -14 \\
\hline & & 30 & 10 & $10 m$ & 26 & 2 & -14 \\
\hline & & 60 & 20 & $20 m$ & 56 & 2 & -14 \\
\hline \multirow[t]{7}{*}{$\mathrm{B}_{\mathrm{II}}$} & $Y^{2} D \bar{D}+X^{2} L \bar{L}$ & 20 & 8 & 8 & 12 & 6 & 18 \\
\hline & $X Y D \bar{D}+X^{2} L \bar{L}$ & 36 & 12 & $8+12 m$ & 8 & 4 & 14 \\
\hline & $Y^{2} Q \bar{Q}+X Y U \bar{U}+X^{2} E \bar{E}$ & 20 & 8 & 12 & 0 & 10 & 50 \\
\hline & $X Y Q \bar{Q}+Y^{2} U \bar{U}+X^{2} E \bar{E}$ & 36 & 12 & $8+12 m$ & 16 & 8 & 46 \\
\hline & $Y^{2} \widehat{Q Q}+X^{2} U \bar{U}+X^{2} E \bar{E}$ & 20 & 8 & 0 & 16 & 8 & 34 \\
\hline & & 30 & 10 & $10 m$ & 16 & 8 & 34 \\
\hline & & 60 & 20 & $20 m$ & 16 & 8 & 34 \\
\hline \multirow[t]{14}{*}{$\mathrm{B}_{\text {III }}$} & none & 36 & 12 & $8+12 m$ & 18 & 3 & 18 \\
\hline & $X^{2} D \bar{D}+X Y L \bar{L}$ & 20 & 8 & 16 & 0 & $\frac{10}{3}$ & $\frac{50}{3}$ \\
\hline & $X Y D \bar{D}+Y^{2} L \bar{L}$ & 20 & 8 & 16 & 4 & $\frac{8}{3}$ & $\frac{34}{3}$ \\
\hline & $X^{2} D \bar{D}+Y^{2} L \bar{L}$ & 28 & 12 & 20 & 8 & $\frac{10}{3}$ & $\frac{38}{3}$ \\
\hline & $X^{2} D \bar{D}+X^{2} L \bar{L}$ & 36 & 12 & $8+12 m$ & 16 & $\frac{10}{3}$ & $\frac{62}{3}$ \\
\hline & $X^{2} Q \bar{Q}+X Y U \bar{U}+X Y E \bar{E}$ & 20 & 8 & 0 & 0 & $\frac{10}{3}$ & $\frac{50}{3}$ \\
\hline & $X^{2} Q \bar{Q}+X^{2} U \bar{U}+X^{2} E \bar{E}$ & 36 & 12 & $8+12 m$ & 12 & 4 & 26 \\
\hline & $Y^{2} \widehat{Q} \bar{Q}+Y^{2} U \bar{U}+Y^{2} E \bar{E}$ & 36 & 12 & $8+12 m$ & 0 & 0 & -6 \\
\hline & $X^{2} Q \bar{Q}+X^{2} U \bar{U}+Y^{2} E \bar{E}$ & 21 & 7 & $1+7 m$ & 18 & 4 & 18 \\
\hline & & 28 & 12 & 8 & 4 & 4 & 18 \\
\hline & & 42 & 14 & $8+14 m$ & 18 & 4 & 18 \\
\hline & $X^{2} Q \bar{Q}+X^{2} U \bar{U}+X Y E \bar{E}$ & 20 & 8 & 8 & 16 & 4 & 22 \\
\hline & & 30 & 10 & $8+10 m$ & 6 & 4 & 22 \\
\hline & & 60 & 20 & $8+20 m$ & 36 & 4 & 22 \\
\hline
\end{tabular}

symmetry with $E=N=0$, resulting in an infinite number of different $Z_{n}$ symmetries that protect it; only the first one (with $n=14$ ) is shown. However, all cases that give $N=0$ are moot for our main purpose in this paper, as there is no reason to forbid high-dimension PQ-violating terms if the strong $C P$ problem cannot be solved anyway.

The examples shown in Tables IX and $\mathrm{X}$ are only some of the many possibilities. There are similar $Z_{n}$ symmetries available for models that have other combinations of vectorlike supermultiplets at both the $\mathrm{TeV}$ and intermediate scales. This is also true of the models with quixes, as, for example, in the last two rows in Table V.

\section{Discrete $R$ symmetries $Z_{n}^{R}$}

Discrete $R$ symmetries provide even more possibilities for an accidental Peccei-Quinn symmetry protected to a high power $p$, including a larger number of cases that satisfy both $A_{2}=A_{3}(\bmod n)$ and $A_{1}=5 A_{3}(\bmod n)$. A nonexhaustive selection of such symmetries for the base models is shown in Table XI. One of the discrete $Z_{24}^{R}$ symmetries had been previously proposed and studied for the MSSM in ${ }^{4}$ Ref. [61] and was found in Refs. [29,64] to extend to base models $\mathrm{B}_{\mathrm{II}}$ and $\mathrm{B}_{\mathrm{III}}$ with suppression $p=10$. There are actually several inequivalent $Z_{24}^{R}$

\footnotetext{
${ }^{4}$ Reference [61] imposed a requirement that the discrete symmetry charges for MSSM quark and lepton superfields respect $S U(5)$ invariance, which limits the possibilities to only a few. We do not impose this requirement. We note that all of the symmetries we find can be made consistent with a partial unification with a Pati-Salam $S U(4)_{\mathrm{PS}} \times S U(2)_{L} \times U(1)_{R}$ [87] embedding, although this is not always immediately obvious with our discrete charge conventions.
} 
TABLE X. Some examples of non- $R$ discrete symmetries $Z_{n}$ satisfying the anomaly cancellation conditions $A_{2}=A_{3}$ (mod $n$ ) and $A_{1}=5 A_{3}(\bmod n)$, obtained from base model $\mathrm{B}_{\mathrm{IV}}$ by adding up to one $\mathbf{5}+\overline{\mathbf{5}}$ or $\mathbf{1 0}+\overline{\mathbf{1 0}}$ of $S U(5)$ at the TeV scale. The meaning of $p$ is that the lowest dimension PQ-violating superpotential term(s) allowed by the discrete symmetry and involving only $X$ and $Y$ are of the form $X^{j} Y^{p-j}$. The next three columns list the charges of $X, Y$, and $H_{u}$, with $m=0,1,2$. The remaining MSSM charges are determined in each case as in Table VII. The last three columns give the Green-Schwarz contribution $\rho_{\mathrm{GS}}$ to the $Z_{n}$ anomalies and the PQ-QCD-QCD and PQ-EM-EM anomalies $N$ and $E$, respectively. The cases with $\rho_{\mathrm{GS}}=0$ do not require a Green-Schwarz mechanism. However, the last two examples shown, with $N=0$, have no PQ-QCD-QCD anomaly and, therefore, have an axionlike particle but do not provide a solution to the strong $C P$ problem.

\begin{tabular}{|c|c|c|c|c|c|c|c|c|c|}
\hline Base & Extension & $n$ & $p$ & $X$ & $Y$ & $H_{u}$ & $\rho_{\mathrm{GS}}$ & $N$ & $3 E$ \\
\hline \multirow[t]{22}{*}{$\mathrm{B}_{\mathrm{IV}}$} & None & 36 & 8 & 3 & 15 & 4 & 18 & 3 & 18 \\
\hline & $X Y D \bar{D}+Y^{2} L \bar{L}$ & 12 & 8 & 1 & 5 & $4 m$ & 0 & 3 & 24 \\
\hline & \multirow[t]{2}{*}{$X Y D \bar{D}+X^{2} L \bar{L}$} & 36 & 8 & 3 & 15 & 8 & 0 & 3 & 12 \\
\hline & & 72 & 14 & 3 & 33 & 2 & 54 & 3 & 12 \\
\hline & $X Y D \bar{D}+X Y L \bar{L}$ & 36 & 8 & 3 & 15 & 4 & 0 & 3 & 18 \\
\hline & $X^{2} D \bar{D}+X^{2} L \bar{L}$ & 36 & 8 & 3 & 15 & 4 & 12 & 2 & 10 \\
\hline & \multirow[t]{3}{*}{$Y^{2} D \bar{D}+X^{2} L \bar{L}$} & 12 & 8 & 1 & 5 & $4 m$ & 8 & 4 & 14 \\
\hline & & 18 & 9 & 1 & 8 & $2+6 m$ & 8 & 4 & 14 \\
\hline & & 36 & 20 & 1 & 17 & $8+12 m$ & 8 & 4 & 14 \\
\hline & \multirow{10}{*}{$X^{2} D \bar{D}+X Y L \bar{L}$} & 12 & 8 & 1 & 5 & $4 m$ & 4 & 2 & 16 \\
\hline & & 14 & 7 & 1 & 6 & 5 & 4 & 2 & 16 \\
\hline & & 16 & 10 & 1 & 7 & 6 & 4 & 2 & 16 \\
\hline & & 18 & 9 & 1 & 8 & $1+6 m$ & 4 & 2 & 16 \\
\hline & & 20 & 12 & 1 & 9 & 8 & 4 & 2 & 16 \\
\hline & & 22 & 11 & 1 & 10 & 9 & 4 & 2 & 16 \\
\hline & & 24 & 14 & 1 & 11 & $2+8 m$ & 4 & 2 & 16 \\
\hline & & 28 & 16 & 1 & 13 & 12 & 4 & 2 & 16 \\
\hline & & 32 & 18 & 1 & 15 & 14 & 4 & 2 & 16 \\
\hline & & 36 & 20 & 1 & 17 & $4+12 m$ & 4 & 2 & 16 \\
\hline & $Y^{2} Q \bar{Q}+Y^{2} U \bar{U}+Y^{2} E \bar{E}$ & 36 & 8 & 3 & 15 & 4 & 0 & 6 & 42 \\
\hline & $X^{2} Q \bar{Q}+X^{2} U \bar{U}+X^{2} E \bar{E}$ & 36 & 8 & 3 & 15 & 4 & 0 & 0 & -6 \\
\hline & $X^{2} Q \bar{Q}+X^{2} U \bar{U}+X Y E \bar{E}$ & 14 & 7 & 1 & 6 & 2 & 0 & 0 & 0 \\
\hline
\end{tabular}

symmetries; the one found in Ref. [61] and applied to $\mathrm{B}_{\mathrm{II}}$ and $B_{I I I}$ in Refs. [29,64] is equivalent (after shifting by a multiple of weak hypercharge) to what we have listed in Table XI in the second row under $\mathrm{B}_{\mathrm{II}}$ and the third row under $\mathrm{B}_{\mathrm{III}}$, with $k=m=0$ and $\rho_{\mathrm{GS}}=18$ in each case. It was also pointed out in Refs. [29,64] that, when this $Z_{24}^{R}$ symmetry is realized in base models $B_{I}$ and $B_{I V}$, it provides only suppression $p=7$; we have chosen not to list these among the examples, although suppression $p=7$ may be good enough if $f_{A}$ is up to $4 \times 10^{9} \mathrm{GeV}$, since it can be promoted to $p=8$ by imposing an extra $Z_{2}$ symmetry that acts only on $X$ and $Y$.

For model $\mathrm{B}_{\mathrm{IV}}$, there are two distinct $Z_{12}^{R}$ symmetries that give $p=7$ (or $p=8$ by the trick just mentioned), one of which requires use of the Green-Schwarz mechanism and one of which does not because $\rho_{\mathrm{GS}}=A_{1}=A_{2}=A_{3}=0(\mathrm{mod}$ 12). For $\rho_{\mathrm{GS}}=0$ with protection up to $p=8$, one also has symmetries with smallest order $n=54$ for models $\mathrm{B}_{\mathrm{I}}, \mathrm{B}_{\mathrm{II}}$, and $\mathrm{B}_{\mathrm{IV}}$ and with protection up to $p=10$ for $n=54$ for models $\mathrm{B}_{\mathrm{III}}$ and $n=108$ for models $\mathrm{B}_{\mathrm{I}}$ and $\mathrm{B}_{\mathrm{IV}}$. There are a plethora of other possibilities beyond those shown in the table.
Adding vectorlike supermultiplets adds to the possibilities for discrete $R$ symmetries. A few examples are shown in Table XII, limited for reasons of brevity to only a subset of the cases that have $\rho_{\mathrm{GS}}=0$, so no Green-Schwarz mechanism necessary, and that have at most one $S U(5)$ multiplet at the $\mathrm{TeV}$ scale.

We have checked that, for every available pair of anomaly coefficients $N$ and $E$ that determine the lowenergy axion phenomenology as discussed in the next section, one can find a variety of corresponding discrete symmetries. From the point of view of low-energy phenomenology, the exact identity of the discrete symmetry may be of limited interest, since it is not possible to determine whether the discrete symmetry is an $R$ symmetry, or its order $n$, or whether it may have $\rho_{\mathrm{GS}}=0$. The more important point seems to be the existence proof that it is always possible to find such discrete symmetries, so that the global PQ symmetry is consistent. The correlation of $N$, $E$, and the possible presence of TeV-scale vectorlike quarks or leptons could eventually point the way to specific ultraviolet completions. 
TABLE XI. Some examples of discrete $R$ symmetries $Z_{n}^{R}$ that satisfy both discrete anomaly cancellation constraints $A_{2}=A_{3}(\bmod n)$ and $A_{1}=5 A_{3}(\bmod n)$ for the base models. The superpotential has charge $2 r$, and gauginos have charge $r$. The meaning of $p$ is that the lowest dimension PQ-violating superpotential term(s) allowed by the discrete symmetry and involving only $X$ and $Y$ are of the form $X^{j} Y^{p-j}$. The next three columns list the charges of $X, Y$, and $H_{u}$ in terms of $k, k^{\prime}=0,1$ and $m=0,1,2$. The remaining MSSM charges are determined in each case as in Table VII. The last column is the Green-Schwarz contribution $\rho_{\mathrm{GS}}$ to the $Z_{n}^{R}$ anomalies. Cases with $\rho_{\mathrm{GS}}=0$ do not use the Green-Schwarz mechanism for anomaly cancellation. The PQ-QCD-QCD and PQ-EM-EM anomalies are $N=3$ and $E=6$ in each case.

\begin{tabular}{|c|c|c|c|c|c|c|c|}
\hline Model & $n$ & $r$ & $p$ & $X$ & $Y$ & $H_{u}$ & $\rho_{\mathrm{GS}}$ \\
\hline \multirow{10}{*}{$\mathrm{B}_{\mathrm{I}}$} & 15 & 1 & 7 & -1 & 5 & $3+5 m$ & 12 \\
\hline & 20 & 1 & 8 & $9+10 k$ & $-5+10 k$ & 13 & 12 \\
\hline & 23 & 1 & 9 & 4 & 13 & 14 & 5 \\
\hline & 30 & 1 & 10 & -1 & 5 & $3+10 m$ & 12 \\
\hline & 35 & 1 & 11 & 4 & 25 & 18 & 17 \\
\hline & 44 & 2 & 12 & 19 & 35 & 42 & 30 \\
\hline & 50 & 1 & 14 & 29 & 15 & 23 & 32 \\
\hline & 54 & 3 & 8 & 39 & -3 & 1 & 0 \\
\hline & 60 & 2 & 16 & 13 & 25 & $6+20 m$ & 54 \\
\hline & 108 & 3 & 10 & $21+18 k+54 k^{\prime}$ & $51+54 k+54 k^{\prime}$ & $7+12 k$ & 0 \\
\hline \multirow[t]{6}{*}{$\mathrm{B}_{\mathrm{II}}$} & 19 & 1 & 7 & -3 & 11 & 0 & 1 \\
\hline & 24 & 1 & 10 & $-1+12 k$ & $5+12 k$ & $1+8 m$ & 18 \\
\hline & 24 & 1 & 10 & $5+12 k$ & $11+12 k$ & $1+8 m$ & 6 \\
\hline & 34 & 1 & 12 & -3 & 11 & -5 & 16 \\
\hline & 54 & 3 & 8 & 9 & 33 & 7 & 0 \\
\hline & 56 & 1 & 18 & $11+28 k$ & $25+28 k$ & 25 & 10 \\
\hline \multirow[t]{7}{*}{$\mathrm{B}_{\mathrm{III}}$} & 13 & 1 & 7 & 6 & -3 & 2 & 8 \\
\hline & 20 & 2 & 8 & 5 & 9 & 6 & 14 \\
\hline & 24 & 1 & 10 & $5+12 k$ & $11+12 k$ & $1+8 m$ & 18 \\
\hline & 24 & 1 & 10 & $-1+12 k$ & $5+12 k$ & $1+8 m$ & 6 \\
\hline & 35 & 1 & 11 & 25 & -3 & 18 & 17 \\
\hline & 48 & 1 & 12 & $19+24 k$ & $41-24 k$ & $1+16 m$ & 6 \\
\hline & 54 & 3 & 10 & $5+12 k$ & $-9+18 k$ & $1+6 k+18 m$ & 0 \\
\hline \multirow[t]{9}{*}{$\mathrm{B}_{\mathrm{IV}}$} & 12 & 1 & 7 & -4 & $5+6 k$ & $1+4 m$ & 0 \\
\hline & 12 & 1 & 7 & $5+6 k$ & -4 & $1+4 m$ & 6 \\
\hline & 16 & 1 & 8 & $5+8 k$ & -4 & 1 & 14 \\
\hline & 20 & 1 & 9 & 12 & $9+10 k$ & 13 & 12 \\
\hline & 25 & 1 & 10 & -3 & 4 & -2 & 7 \\
\hline & 28 & 1 & 11 & $4+14 k$ & $11+14 k^{\prime}$ & -3 & 24 \\
\hline & 32 & 1 & 12 & $5+16 k$ & -4 & 17 & 30 \\
\hline & 54 & 3 & 8 & $-9+18 k$ & $39+9 k$ & $1+6 k$ & 0 \\
\hline & 108 & 3 & 10 & 90 & $21+54 k$ & 7 & 0 \\
\hline
\end{tabular}

\section{Impact on baryon number and lepton number violation}

Recall that in the MSSM there are renormalizable superpotential terms that violate lepton number $\mathrm{L}$ and baryon number B, schematically:

$$
\begin{aligned}
W_{\mathrm{L}-\text { violating }} & =H_{u} \ell+q \ell \bar{d}+\ell \ell \bar{e}, \\
W_{\text {B-violating }} & =\bar{u} \bar{d} \bar{d} .
\end{aligned}
$$

Taken together, these would predict very rapid proton decay. The most common way of avoiding this is to impose the $Z_{2}$ matter parity matter parity $=(-1)^{3(\mathrm{~B}-\mathrm{L})}$ discrete symmetry (or, equivalently, $R$ parity) [88-90]. There are also nonrenormalizable operators that suppressed by the cutoff scale but violate both $\mathrm{B}$ and $\mathrm{L}$ and so could directly mediate proton decay in violation of current bounds:

$$
W=\frac{1}{M_{P}} q q q \ell+\frac{1}{M_{P}} \bar{u} \bar{u} \bar{d} \bar{e} .
$$

As was noted in Ref. [60] for the $Z_{22}$ discrete symmetry example found there and in Ref. [61] for their $Z_{24}^{R}$ symmetry, the discrete symmetry that protects the PQ symmetry can also help by forbidding these dangerous baryon-number- and lepton-number-violating operators.

In Table XIII, we show the discrete symmetry charges $z_{\mathcal{O}}-2 r$ of the above superpotential operators $\mathcal{O}$. The operator is allowed if the entry in the table vanishes. 
TABLE XII. Some examples of discrete $R$ symmetries $Z_{n}^{R}$ that satisfy the anomaly cancellation conditions $A_{1}=A_{2}=A_{3}=0(\bmod n)$ without the Green-Schwarz mechanism (so $\rho_{\mathrm{GS}}=0$ ). The superpotential has charge $2 r$, and gauginos have charge $r$. The meaning of $p$ is that the lowest dimension PQ-violating superpotential term(s) allowed by the discrete symmetry and involving only $X$ and $Y$ are of the form $X^{j} Y^{p-j}$. The next three columns list the charges of $X, Y$, and $H_{u}$, in terms of $k=0,1, m=0,1,2$, and $s=0,1,2,3$. The remaining MSSM charges are determined in each case as in Table VII. The last two columns give the PQ-QCD-QCD and PQ-EM-EM anomalies $N$ and $E$, respectively.

\begin{tabular}{|c|c|c|c|c|c|c|c|c|c|}
\hline Base & Extension & $n$ & $r$ & $p$ & $X$ & $Y$ & $H_{u}$ & $N$ & $3 E$ \\
\hline \multirow[t]{3}{*}{$\mathrm{B}_{\mathrm{I}}$} & None & 54 & 3 & 8 & 39 & -3 & 1 & 3 & 18 \\
\hline & $X^{2} D \bar{D}+X Y L \bar{L}$ & 22 & 1 & 8 & 9 & -3 & 9 & 4 & 14 \\
\hline & $X Y D \bar{D}+Y^{2} L \bar{L}$ & 24 & 1 & 8 & $7+12 k$ & $5+12 k$ & $7+8 m$ & 2 & -2 \\
\hline \multirow[t]{6}{*}{$\mathrm{B}_{\mathrm{II}}$} & None & 54 & 3 & 8 & 9 & 33 & 7 & 3 & 18 \\
\hline & $X^{2} D \bar{D}+Y^{2} L \bar{L}$ & 20 & 2 & 8 & 5 & 9 & 14 & 2 & 34 \\
\hline & $Y^{2} D \bar{D}+X^{2} L \bar{L}$ & 32 & 1 & 10 & $3+16 k$ & $-7+16 k$ & 5 & 6 & 18 \\
\hline & $Y^{2} D \bar{D}+X Y L \bar{L}$ & 108 & 6 & 20 & 11 & 87 & $22+36 m$ & 6 & 30 \\
\hline & $Y^{2} Q \bar{Q}+X^{2} U \bar{U}+X^{2} E \bar{E}$ & 24 & 1 & 10 & $5+6 s$ & $11+6 s$ & $5+8 m$ & 8 & 34 \\
\hline & $Y^{2} Q \bar{Q}+X^{2} U \bar{U}+Y^{2} E \bar{E}$ & 56 & 1 & 18 & $11+14 s$ & $25+14 s$ & -3 & 8 & 58 \\
\hline \multirow[t]{5}{*}{$\mathrm{B}_{\mathrm{III}}$} & None & 54 & 3 & 10 & $5+12 k$ & $-9+18 k$ & $1+6 k+18 m$ & 3 & 18 \\
\hline & $X Y D \bar{D}+X Y L \bar{L}$ & 20 & 2 & 8 & 5 & 9 & 6 & $\frac{8}{3}$ & $\frac{46}{3}$ \\
\hline & $X^{2} D \bar{D}+X^{2} L \bar{L}$ & 24 & 1 & 8 & $9+12 k$ & $-1+12 k$ & $1+8 m$ & $\frac{10}{3}$ & $\frac{62}{3}$ \\
\hline & $X^{2} Q \bar{Q}+Y^{2} U \bar{U}+X^{2} E \bar{E}$ & 24 & 1 & 10 & $5+6 s$ & $11+6 s$ & $5+8 m$ & $\frac{8}{3}$ & $\frac{46}{3}$ \\
\hline & $X^{2} Q \bar{Q}+Y^{2} U \bar{U}+Y^{2} E \bar{E}$ & 56 & 1 & 18 & $11+14 s$ & $25+14 s$ & -3 & $\frac{3}{3}$ & $\frac{32}{3}$ \\
\hline \multirow[t]{8}{*}{$\mathrm{B}_{\mathrm{IV}}$} & None & 12 & 1 & 7 & -4 & $5+6 k$ & $1+4 m$ & 3 & 18 \\
\hline & & 54 & 3 & 8 & $-9+18 k$ & $39+9 k$ & $1+6 k$ & 3 & 18 \\
\hline & & 108 & 3 & 10 & 90 & $21+54 k$ & 7 & 3 & 18 \\
\hline & $Y^{2} D \bar{D}+X^{2} L \bar{L}$ & 12 & 2 & 8 & 5 & 9 & $2+4 m$ & 4 & 14 \\
\hline & $X^{2} D \bar{D}+Y^{2} L \bar{L}$ & 16 & 1 & 8 & -4 & $5+8 k$ & 3 & 2 & 22 \\
\hline & $X Y Q \bar{Q}+Y^{2} U \bar{U}+Y^{2} E \bar{E}$ & 24 & 4 & 10 & 5 & $-1+12 k$ & $2+4 k+8 m$ & 4 & 32 \\
\hline & $Y^{2} \widehat{Q Q}+X^{2} U \bar{U}+X^{2} E \bar{E}$ & 28 & 1 & 11 & $11+7 s$ & $4-7 s+14 k$ & 11 & 4 & 14 \\
\hline & $Y^{2} Q \bar{Q}+X Y U \bar{U}+X^{2} E \bar{E}$ & 60 & 2 & 16 & 7 & -5 & $2+20 m$ & 5 & 22 \\
\hline
\end{tabular}

Note that the lepton-number-violating operator $H_{u} \ell$ is always forbidden if the discrete symmetry is an $R$ symmetry but is always allowed if it is a non- $R$ symmetry (with $r=0$ ). This is because we required that the square of this operator is allowed to provide neutrinos masses. Also, the discrete symmetry always forbids the operators $q \ell \bar{d}$ and $\ell \ell \bar{e}$. This is because, if they are allowed, one can check that

TABLE XIII. The discrete charges $z_{\mathcal{O}}-2 r$ for B- and/or Lviolating operators $\mathcal{O}$ in the MSSM superpotential, for each of the base models (and their extensions), in our conventions. Each operator is allowed only if the entry vanishes. The terms $\ell \ell \bar{e}$ and $q \ell \bar{d}$ are, therefore, always forbidden, because otherwise the PQviolating term $Y^{4}$ would be allowed. The term $H_{u} \ell$ is forbidden for discrete $R$ symmetries $(r \neq 0)$.

\begin{tabular}{lccc}
\hline \hline $\mathcal{O}$ & $\mathrm{B}_{\mathrm{I}}$ & $\mathrm{B}_{\mathrm{II}}, \mathrm{B}_{\mathrm{IV}}$ & $\mathrm{B}_{\mathrm{III}}$ \\
\hline$H_{u} \ell$ & $-r$ & $-r$ & $-r$ \\
$\ell \ell \bar{e}, q \ell \bar{d}$ & $-2 x+r$ & $2 x-r$ & $-6 x+3 r$ \\
$\bar{u} \bar{d} \bar{d}$ & $h-4 x+4 r$ & $h+4 x$ & $h-12 x+8 r$ \\
$q q q \ell$ & $-h-r$ & $-h-r$ & $-h-r$ \\
$\bar{u} \bar{u} \bar{d} \bar{e}$ & $h-4 x+5 r$ & $h+4 x+r$ & $h-12 x+9 r$ \\
\hline \hline
\end{tabular}

the term $Y^{4}$ would also be allowed and would violate the PQ symmetry, against our defining requirement for the discrete symmetry. Therefore, the PQ-protecting $Z_{n}^{R}$ discrete symmetry never allows renormalizable $\mathrm{L}$ violation, and $Z_{n}$ allows only soft $\mathrm{L}$ violation. Regarding the other Band L-violating operators, the charges in the table have no particular reason to vanish, and we find that in the vast majority of discrete symmetry cases they do not. A catalog of specific cases will not be attempted here, but the general lesson is that quite often the discrete symmetries are powerful enough to automatically suppress proton decay sufficiently to satisfy present bounds, in addition to providing high-quality PQ symmetry. One can also always supplement the PQ-protecting discrete symmetry with either matter parity or the $Z_{3}$ baryon triality [82], which forbids all proton decay.

\section{AXION SIGNALS AND DETECTION PROSPECTS}

In this section, we discuss the low-energy axion couplings to photons, electrons, and nucleons, and their impact on limits and detection prospects, for the supersymmetric 
base models and their extensions discussed above. For comparison, we will also give results for the standard nonsupersymmetric benchmark QCD axion models of Kim-Shifman-Vainshtein-Zakharov (KSVZ) [6,7] and DFSZ [8,9] of types I and II.

In the nonsupersymmetric benchmark DFSZ-I model, the Standard Model leptons and down-type quarks get their mass from one Higgs doublet, and the up-type quarks couple to the other Higgs doublet, as is the case with the MSSM. In DFSZ-II, the Standard Model leptons instead couple to the Higgs doublet that provides for the mass of the up-type quarks. In the KSVZ models, the Standard Model quarks and leptons are not charged under $U(1)_{\mathrm{PQ}}$, unlike the DFSZ models. We consider several KSVZ constructions in which the heavy vectorlike quarks mediating the PQ anomaly have different electroweak quantum numbers. The original Kim model [6], in which the vectorlike quark transforms under the Standard Model gauge group as $(\mathbf{3}, \mathbf{1}, 0)+(\overline{\mathbf{3}}, \mathbf{1}, 0)$, will be called $\mathrm{KSVZ}_{0}$, while $\mathrm{KSVZ}_{D}, \mathrm{KSVZ}_{U}$, and $\mathrm{KSVZ}_{Q}$ have vectorlike quarks with gauge quantum numbers as that of $D+\bar{D}$, $U+\bar{U}$, and $Q+\bar{Q}$, respectively. Table XIV summarizes these benchmarks, with their PQ-QCD-QCD anomaly coefficients $N$ and the coupling coefficients defined in Eqs. (2.20) and (2.21) and appearing in Eqs. (2.27)-(2.32) and (2.34). Among these benchmarks models, only $\mathrm{KSVZ}_{0}$, $\mathrm{KSVZ}_{D}$, and $\mathrm{KSVZ}_{U}$ models have domain wall number $N_{\mathrm{DW}}=1$.

As noted in Ref. [91], all four supersymmetric DFSZ base models $\mathrm{B}_{\mathrm{I}}, \mathrm{B}_{\mathrm{II}}, \mathrm{B}_{\mathrm{III}}$, and $\mathrm{B}_{\mathrm{IV}}$ have $E / N=2$, which is close to canceling the model-independent contribution $-1.92 \pm 0.04$ in Eqs. (2.27) and (2.49), and so have suppressed axion-photon couplings compared to the standard nonsupersymmetric benchmark cases. However, as we

TABLE XIV. Benchmark nonsupersymmetric QCD axion models and the fermions that mediate the $U(1)_{\mathrm{PQ}}$ anomaly. $N$ is the $U(1)_{\mathrm{PQ}}-\left[S U(3)_{c}\right]^{2}$ anomaly coefficient, and $c_{\gamma}, c_{u}, c_{d}$, and $c_{e}$ are low-energy axion coupling coefficients for photons, up-type quarks, down-type quarks, and electrons, respectively, appearing in Eqs. (2.27)-(2.32) and (2.34). In KSVZ constructions, only the new vectorlike quarks carry a PQ charge. In both DFSZ models, $\tan \beta=s_{\beta} / c_{\beta}$ is the ratio of the Higgs VEVs analogous to the MSSM. The domain wall number is $N_{\mathrm{DW}}=2 \mathrm{~N}$ in all of these benchmark cases.

\begin{tabular}{lcccccc}
\hline \hline Benchmark & PQ charged fermions & $N$ & $c_{\gamma}$ & $c_{u}$ & $c_{d}$ & $c_{e}$ \\
\hline $\mathrm{KSVZ}_{0}$ & $(\mathbf{3}, \mathbf{1}, 0)+(\overline{\mathbf{3}}, \mathbf{1}, 0)$ & $\frac{1}{2}$ & 0 & 0 & 0 & 0 \\
$\mathrm{KSVZ}_{D}$ & $D+\bar{D}$ & $\frac{1}{2}$ & $\frac{2}{3}$ & 0 & 0 & 0 \\
$\mathrm{KSVZ}_{U}$ & $U+\bar{U}$ & $\frac{1}{2}$ & $\frac{8}{3}$ & 0 & 0 & 0 \\
$\mathrm{KSVZ}_{Q}$ & $Q+\bar{Q}$ & 1 & $\frac{5}{3}$ & 0 & 0 & 0 \\
$\mathrm{DFSZ}_{-\mathrm{I}}$ & $\mathrm{SM} \mathrm{fermions}$ & 3 & $\frac{8}{3}$ & $\frac{c_{\beta}^{2}}{3}$ & $\frac{s_{\beta}^{2}}{3}$ & $\frac{s_{\beta}^{2}}{3}$ \\
DFSZ-II & SM fermions & 3 & $\frac{2}{3}$ & $\frac{c_{\beta}^{2}}{3}$ & $\frac{s_{\beta}^{2}}{3}$ & $-\frac{c_{\beta}^{2}}{3}$ \\
\hline \hline
\end{tabular}

will see below, adding extra vectorlike supermultiplets that get their masses from the VEVs of the $X$ and $Y$ scalar fields can enhance the axion couplings. This is because they have nonzero net PQ charges contributing to the anomaly coefficients $N$ and $E$. Interestingly, the extensions with $N_{\text {DW }}=1$ that avoid the cosmological domain wall problem have the smallest $|N|$ and, therefore, can give rise to the most enhanced low-energy axion couplings. As discussed in Sec. III, for every $(N, E)$ found in the base models and their extensions, the PQ symmetry can be protected to a high degree (generally in a variety of different ways). Consequently, high-quality QCD axions in many of these extensions, including some $N_{\mathrm{DW}}>1$ cases, should be accessible to future axion searches.

We begin with the axion coupling to electrons, because this gives the most robust bound on $f_{A}$ from present astrophysical data. Figure 2 shows the axion-electron coupling $\left|g_{A e}\right|$ as a function of the axion mass $m_{A}$ [and, equivalently, $f_{A}$, through Eq. (2.26)], for large $\tan \beta$. For the DFSZ-I benchmark model and all supersymmetric models,

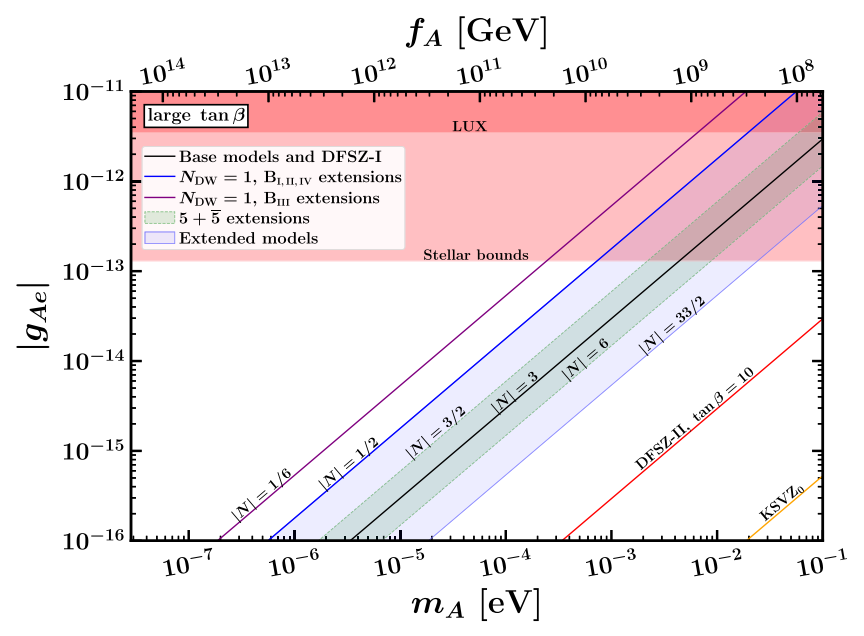

FIG. 2. The axion-electron coupling $\left|g_{A e}\right|$ as a function of the axion mass $m_{A}$ and the axion decay constant $f_{A}$. The results at large $\tan \beta$ for the four supersymmetric base models, and the DFSZ-I benchmark, all lie on the $|N|=3$ line, where $N$ is the PQ-QCD-QCD anomaly. The range for base models extended by only a single $\mathbf{5}+\overline{\mathbf{5}}$ occupy the green shaded band bounded by $|N|=3 / 2$ and $|N|=6$. The light blue region is the allowed range for extensions of base models that include a $\mathbf{5}+\overline{\mathbf{5}}$ or $\mathbf{1 0}+\overline{\mathbf{1 0}}$ at the TeV scale and/or $\mathbf{5}+\overline{\mathbf{5}}$ or $\mathbf{1 0}+\overline{\mathbf{1 0}}$ at $M_{\text {int }}$, or $2 \times(L+\bar{L})$ at the TeV scale and an exotic quix pair $D_{6}+\bar{D}_{6}$ at $M_{\text {int }}$. The cases with $N_{\mathrm{DW}}=1$ have either $|N|=1 / 2$ (extensions of $\mathrm{B}_{\mathrm{I}, \mathrm{II}, \mathrm{IV}}$, heavier solid blue line) or $1 / 6$ (extensions of $\mathrm{B}_{\mathrm{III}}$, solid purple line) and saturate the upper limit on $\left|g_{A e}\right|$ in these extensions. The shading for allowed supersymmetric models excludes $1 / 6<|N|<1 / 2$, where there are no cases. The much smaller predictions of the benchmarks DFSZ-II with $\tan \beta=10$ and $\mathrm{KSVZ}_{0}$ are also shown for comparison. The current experimental limits on $\left|g_{A e}\right|$ from the LUX experiment and from stellar cooling are also shown on the plot, as labeled. 
the results are not very sensitive to the value of $\tan \beta$ as long as it is large, and $\left|g_{A e}\right|=m_{e} /|N| f_{A}$ in the large $\tan \beta$ limit, so the lines can be simply labeled by $|N|$. The line $|N|=3$ is, thus, the common result for DFSZ-I and for all four base models. It is surrounded by a green shaded region bounded by the labels $|N|=3 / 2$ and $|N|=6$, which is the range of possibilities for all extensions of the base models with only one $\mathbf{5}+\overline{\mathbf{5}}$ at either the intermediate or $\mathrm{TeV}$ scale. The larger blue shaded band between $|N|=1 / 2$ and $|N|=33 / 2$ is the range of results for the more general class of models obtained by extending the base models to include $\mathbf{5}+\overline{\mathbf{5}}$ or $\mathbf{1 0}+\overline{\mathbf{1 0}}$ at the TeV scale and/or $\mathbf{5}+\overline{\mathbf{5}}$ or $\mathbf{1 0}+\overline{\mathbf{1 0}}$ at $M_{\text {int }}$, or $2 \times(L+\bar{L})$ at the $\mathrm{TeV}$ scale and an exotic quix pair $D_{6}+$ $\bar{D}_{6}$ at $M_{\text {int }}$. The largest possible couplings $g_{A e}$ for a given $f_{A}$ are obtained for $|N|=1 / 6$ for extensions of $\mathrm{B}_{\mathrm{III}}$ and $|N|=1 / 2$ for extensions of $\mathrm{B}_{\mathrm{I}, \mathrm{II}, \mathrm{IV}}$. There are no extensions that have $|N|$ in between the allowed values $1 / 2$ and $1 / 6$, so we did not include that range in the blue shaded band. We also show the results for $\mathrm{KSVZ}_{0}$ and DFSZ-II with $\tan \beta=10$. Note that $\left|g_{A e}\right|$ is much smaller, and more sensitive to large $\tan \beta$, in DFSZ-II than in DFSZ-I. In these cases, since the tree-level $g_{A e}$ is zero and very small, respectively, we include the leading log renormalization contribution from Eq. (2.32).

The models with $N_{\text {DW }}=1$ saturate the upper limit on the axion-electron coupling as shown in the figure. These include extensions of base models $\mathrm{B}_{\mathrm{I}}, \mathrm{B}_{\mathrm{II}}$, and $\mathrm{B}_{\mathrm{IV}}$ with $|N|=1 / 2$ and $\mathrm{B}_{\text {III }}$ with $|N|=1 / 6$ shown in Table V. There are also extensions of base model $\mathrm{B}_{\text {III }}$ that have $|N|=1 / 2$ but with $N_{\mathrm{DW}} \neq 1$. The lower limits in all extensions correspond to the models with largest $|N|$ that can occur. In all base model extensions with $S U(5)$ pairs, or $D_{6}+\bar{D}_{6}$ quixes, the largest $|N|=33 / 2$ occurs in $\mathrm{B}_{\text {II }}$ extended with a $\mathbf{1 0}+\overline{\mathbf{1 0}}$ at the TeV scale and a $\mathbf{1 0}+\overline{\mathbf{1 0}}$ at $M_{\text {int }}$. And, in the extensions with exactly one $\mathbf{5}+\overline{\mathbf{5}}$, the largest $|N|=6$ occurs in the extension of $\mathrm{B}_{\text {II }}$ with a $\mathbf{5}+\overline{\mathbf{5}}$ at the TeV scale.

Current experimental limits from the LUX experiment [92] and much stronger bounds from the brightness of the tip of the red-giant branch $[93,94]$ are shown as shaded regions in Fig. 2. There are also bounds [95-97] from the cooling of white dwarfs, which are somewhat less strong than the red-giant bounds, and some hints of stellar cooling that might be ascribed to axions [98]. The red-giant bound on the axion-electron coupling $g_{A e}<1.3 \times 10^{-13}$ sets the most stringent astrophysical constraint throughout our supersymmetric DFSZ axion model space:

$$
f_{A}>\frac{\sin ^{2} \beta}{|N|}\left(3.9 \times 10^{9} \mathrm{GeV}\right),
$$

which can also be written directly in terms of the $X, Y$ scalar field VEVs [see Eq. (2.43)] as

$$
\left|v_{A}\right|>\sin ^{2} \beta\left(7.8 \times 10^{9} \mathrm{GeV}\right) \text {. }
$$

The lower bound on the axion decay constant for $|N|=$ $(1 / 6,1 / 2,3)$ for large $\tan \beta$ is $f_{A} \gtrsim\left(2.3 \times 10^{10}, 7.8 \times 10^{9}\right.$, $\left.1.3 \times 10^{9}\right) \mathrm{GeV}$, which corresponds to an upper bound on the axion mass $m_{A} \lesssim\left(2.4 \times 10^{-4}, 7.3 \times 10^{-4}, 4.4 \times 10^{-3}\right) \mathrm{eV}$.

We now turn to the axion-photon coupling and direct detection prospects for axions. In Fig. 3, we show the axion-photon coupling $\left|g_{A \gamma}\right|$ as a function of the axion mass
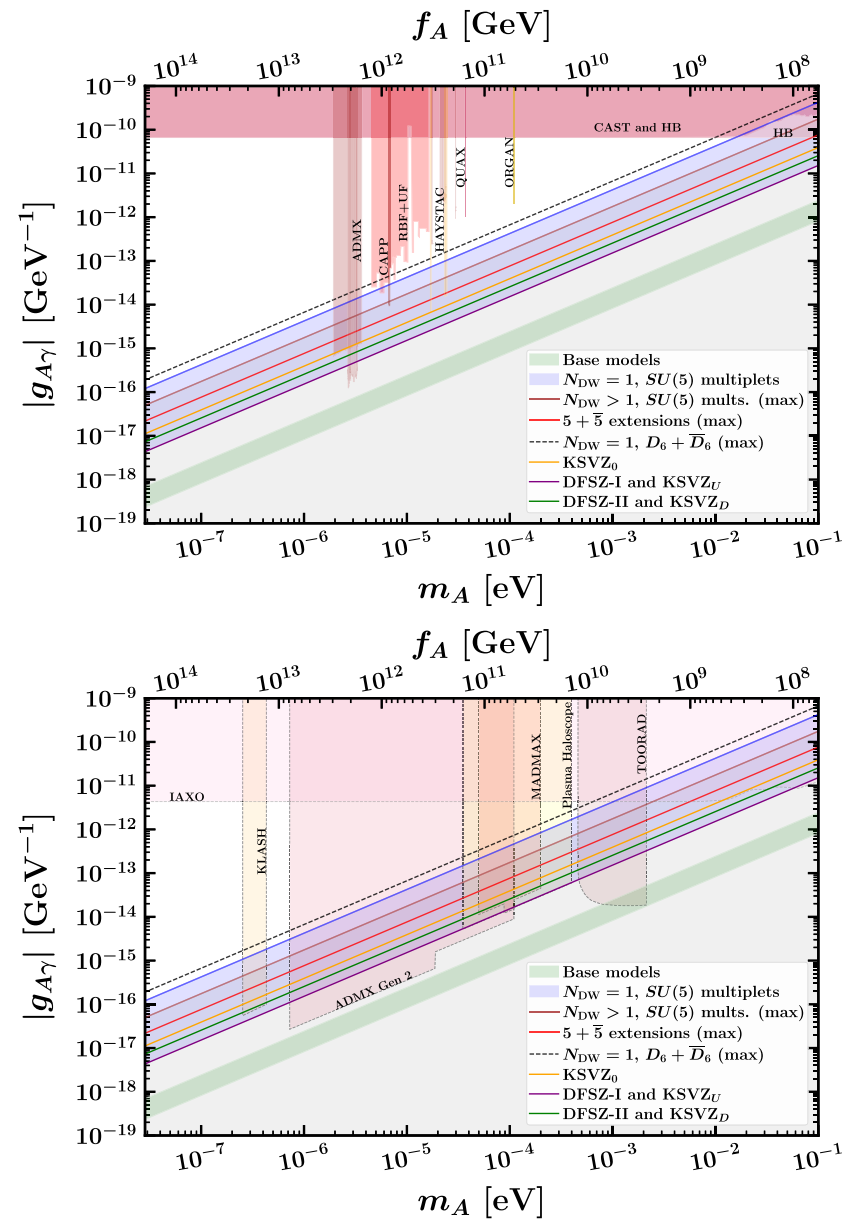

FIG. 3. Model predictions for axion-photon coupling $g_{A \gamma}$ as a function of the axion mass and the axion decay constant, with current bounds from various axion searches (top panel) and projections for future experiments (bottom panel). The prediction, including uncertainty, for supersymmetric DFSZ base models is the shaded green band. Cases with $N_{\mathrm{DW}}=1$ that occur in base models extended to include $S U(5)$ multiplets are shown as the shaded blue region. The largest $\left|g_{A \gamma}\right|$ that have $N_{\text {DW }}>1$ in such extensions is the solid brown line, and the maximum in extensions that include only a single $\mathbf{5}+\overline{\mathbf{5}}$ is the solid red line. The largest coupling found in models with $2 \times(L+\bar{L})$ at the TeV scale and a quix pair $D_{6}+\bar{D}_{6}$ at $M_{\text {int }}$ is the dashed black line. The light gray shading everywhere below the brown line is to emphasize that the axion can accidentally decouple from the photon in some extensions with $N_{\text {DW }}>1$. For comparison, the lines for the standard benchmark axion models $\mathrm{KSVZ}_{0}$, DFSZ-I (the same as $\mathrm{KSVZ}_{U}$ ), and DFSZ-II (the same as $\mathrm{KSVZ}_{D}$ ) are shown. 
$m_{A}$ and the axion decay constant $f_{A}$. The axion-photon coupling depends only on the ratio $E / N$ and $f_{A}$, so each line in this plot corresponds to a value of $|E / N-1.92(4)|$. The results for the base models (which have $E / N=2$ ), including the uncertainty on the model-independent contribution, are shown as the green shaded band. The blue shaded region shows the range of much larger couplings obtained for extended models made from $S U(5)$ multiplet combinations that give $N_{\mathrm{DW}}=1$. The $E / N$ values that occur in these extensions were listed in Table V. This blue shaded region is bounded from above by a solid blue line that corresponds to the extensions that have $E / N=68 / 3$, and the lower bound for these models is $E / N=8 / 3$ (which is the same as DFSZ-I). This shows that requiring $N_{\mathrm{DW}}=1$ guarantees that the axion-photon coupling is at least as large as for the DFSZ-I model, and is usually considerably larger, for a given axion mass. The extensions of base models with $2 \times(L+\bar{L})$ at the $\mathrm{TeV}$ scale and a quix pair $D_{6}+\bar{D}_{6}$ at $M_{\text {int }}$ can give an even larger axion-photon coupling compared to the extensions with $S U(5)$ multiplets. This is shown by the dashed black line that corresponds to $E / N=104 / 3$, which occurs in the extension of B II with $N_{\text {DW }}=1$.

Also shown in Fig. 3 is a light gray shaded region obtained for models with $N_{\text {DW }}>1$, obtained by extending the base models to include a $\mathbf{5}+\overline{\mathbf{5}}$ or $\mathbf{1 0}+\overline{\mathbf{1 0}}$ at the $\mathrm{TeV}$ scale and/or $\mathbf{5}+\overline{\mathbf{5}}$ or $\mathbf{1 0}+\overline{\mathbf{1 0}}$ at $M_{\text {int }}$. The upper limit in these models with $N_{\mathrm{DW}}>1$, shown with a solid brown line, is realized by an extension with a $\mathbf{1 0}+\overline{\mathbf{1 0}}$ at the $\mathrm{TeV}$ scale and another $\mathbf{1 0}+\overline{\mathbf{1 0}}$ at $M_{\text {int }}$ with $E / N=-20 / 3$, $N_{\text {DW }}=3$. The base models extended with only a $\mathbf{5}+\overline{\mathbf{5}}$ at the TeV scale or $M_{\text {int }}$ cannot have $N_{\mathrm{DM}}=1$ and have an upper limit for $\left|g_{A \gamma}\right|$ shown as the solid red line in Fig. 3. This corresponds to $E / N=17 / 3$, realized in an extension of the model $\mathrm{B}_{\mathrm{II}}$ with a $\mathbf{5}+\overline{\mathbf{5}}$ at the TeV scale. The axionphoton coupling can vanish within errors in some of the extensions (including quixotic extensions) of base models when $E / N$ happens to be close to the imperfectly known model-independent contribution $-1.92 \pm 0.04$, as will be further illustrated below in the bottom panel in Fig. 4. This is the reason for shading the entire region in light gray below the solid brown line. Also shown in Fig. 3 are the lines for the nonsupersymmetric DFSZ-I, DFSZ-II, and $\mathrm{KSVZ}_{0}$ benchmark models. Note that, as can be seen in Table XIV, DFSZ-I and DFSZ-II have the same axionphoton couplings as $\mathrm{KSVZ}_{U}$ and $\mathrm{KSVZ}_{D}$, respectively.

The current experimental bounds on $g_{A \gamma}$ as a function of $m_{A}$ are shown as shaded regions in the top panel in Fig. 3. These include limits from the helioscope CAST $[99,100]$ and from evolution of horizontal branch stars [101]. By coincidence, these happen to give almost the same limit $g_{A \gamma}<6.5 \times 10^{-11} \mathrm{GeV}^{-1}$ over a very wide range of $f_{A}$, although the CAST bound becomes weaker for $f_{A} \lesssim 4 \times 10^{8} \mathrm{GeV}$. Also shown are the results of searches
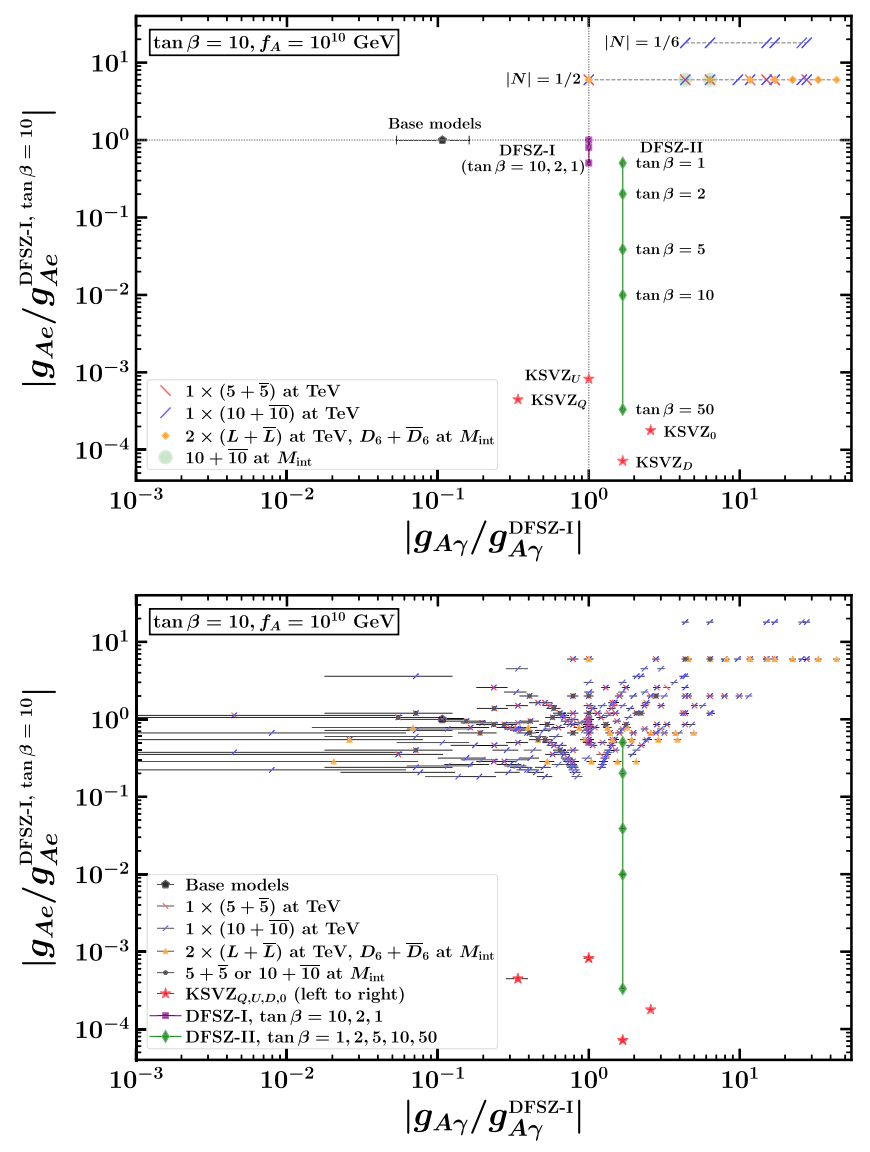

FIG. 4. The axion-electron coupling plotted against the axionphoton coupling, both rescaled by the corresponding couplings of the benchmark nonsupersymmetric DFSZ-I model with $\tan \beta=$ 10 and $f_{A}=10^{10} \mathrm{GeV}$. Different points on the plot correspond to the base models and their extensions, categorized by the additional particle content at the $\mathrm{TeV}$ scale as discussed in the text, again with fixed $\tan \beta=10$. The top panel shows only the models with $N_{\mathrm{DW}}=1$ in these extensions, while the bottom panel shows all possible combinations of mass terms for extra vectorlike supermultiplets in each model extension, as labeled. Extensions with $N_{\mathrm{DW}}=1$ (in the top-right corner in both panels) have axion-photon and axion-electron couplings both enhanced. Also shown in both panels are the points for the benchmarks $\mathrm{KSVZ}_{Q, U, D, 0}$ (independent of $\tan \beta$ ), DFSZ-I, and DFSZ-II for various choices of $\tan \beta$, as labeled. The uncertainty bars on $g_{A \gamma}$ are shown for the base models in the top panel and for all points in the bottom panel.

over much narrower bands in $f_{A}$ by haloscopes, under the assumption that axions are the dark matter: ADMX [102-105], CAPP [106], RBF [107], UF [108], HAYSTAC [109,110], QUAX [111], and ORGAN [112]. (In making Figs. 3 and 5, we made substantial use of the axion limit data collected in Ref. [113].) This shows that some limited ranges of $f_{A}$ for extended supersymmetric models with $N_{\text {DW }}=1$ have already been probed. In the lower panel in Fig. 3, the shaded regions with dashed borders show the projections for future sensitivity from the helioscope IAXO [114] and haloscopes (ADMX [115], KLASH [116], 


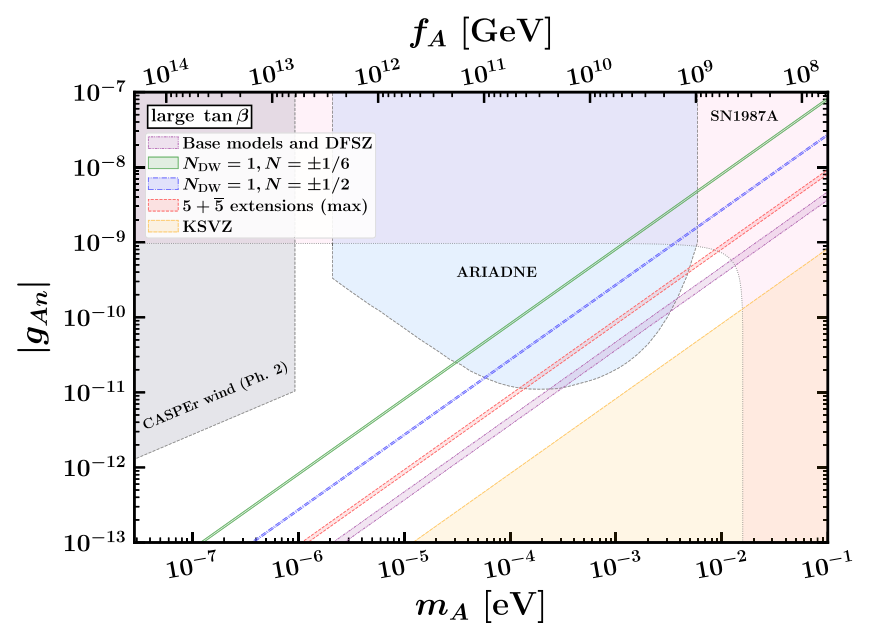

FIG. 5. The axion-neutron coupling as a function of the axion mass and the axion decay constant. The model predictions for large $\tan \beta$ are diagonal bands including uncertainties in the coupling. As labeled, they correspond to the supersymmetric base models (the same as nonsupersymmetric DFSZ-I and DFSZ-II benchmarks), the extensions with $N_{\mathrm{DW}}=1$ that can have enhanced axion-nucleon couplings, and the case that gives rise to the largest axion-nucleon coupling in the extension with a single $\mathbf{5}+\overline{\mathbf{5}}$. Within uncertainties, the neutron may decouple from KSVZ axions, as emphasized by the orange shading extending to very low couplings. The experimental bound from supernova SN 1987A is also shown, as it applies to the supersymmetric DFSZ models as parameterized by Eqs. (2.51) and (2.52) with large $\tan \beta$, taking into account the correlation with $g_{A p}$. Also shown are future projections for CASPEr wind (phase 2) and ARIADNE. The projection for the ARIADNE experiment is based on an optimistic choice regarding the $C P$-violating coupling of axions to neutrons, as discussed in the text.

MADMAX [117], plasma haloscope [118], and TOORAD [119]). Except for a narrow range in the case of TOORAD, these do probe the supersymmetric DFSZ base models, but it is encouraging that the haloscopes do cover many of the possibilities for the model extensions, including essentially all of the allowed range predicted by our models with $N_{\text {DW }}=1$. However, it is again important to note that the haloscope search projections assume that axions are the main component of the dark matter.

The axion couplings to electrons and photons both scale with $1 / f_{A}$. It is, therefore, interesting to compare the ratios $g_{A e} / g_{A e}^{\text {DFSZ-I,tan } \beta=10}$ and $g_{A \gamma} / g_{A \gamma}^{\text {DFSZ-I }}$, in which the dependence on the scale $f_{A}$ (and the axion mass) very nearly cancels. We choose the normalizing denominators to be the results for the nonsupersymmetric benchmark DFSZ-I (with $\tan \beta=10$ ). Figure 4 shows a scatter plot of these ratios for the base models and their extensions along with the benchmarks DFSZ-I, DFSZ-II, and $\mathrm{KSVZ}_{Q, U, D, 0}$, as labeled. In the plot, the extensions of base models are categorized based on the additional particle content at the $\mathrm{TeV}$ scale, which could eventually be discovered in collider experiments. First, there are models with a $\mathbf{5}+\overline{\mathbf{5}}$ or $\mathbf{1 0}+\overline{\mathbf{1 0}}$ at the $\mathrm{TeV}$ scale with or without a $\mathbf{5}+\overline{\mathbf{5}}$ or $\mathbf{1 0}+$ $\overline{\mathbf{1 0}}$ at $M_{\text {int }}$, then there are models with two pairs of vectorlike leptons $L+\bar{L}$ at the $\mathrm{TeV}$ scale and $D_{6}+\bar{D}_{6}$ quixes at $M_{\text {int }}$, and lastly there are models with no new particle content at the $\mathrm{TeV}$ scale which includes the extensions with a $\mathbf{5}+\overline{\mathbf{5}}$ or $\mathbf{1 0}+\overline{\mathbf{1 0}}$ at $M_{\text {int }}$. In these extensions, different combinations of the superpotential mass terms involving the $X$ and $Y$ fields for the additional vectorlike supermultiplets give rise to different points in the figure. In the top panel in Fig. 4, we show only cases with $N_{\text {DW }}=1$, whereas in the bottom panel we show all possibilities (i.e., $N_{\mathrm{DW}} \geq 1$ ) that occur in these extensions. Also, in the top panel, the uncertainty bars are shown only for the base models, for which the uncertainties are significant due to the proximity of $E / N=2$ to 1.92(4). In the bottom panel, the $g_{A \gamma}$ uncertainty bars are shown for all points.

Except for the $f_{A}$ dependence that enters the ratio $g_{A e} / g_{A e}^{\text {DFSZ-I,tan } \beta=10}$ through $\Delta C_{A e}$ for models in which $C_{A e}$ vanishes at tree level, the plots in Fig. 4 do not otherwise depend on the axion decay constant (or, equivalently, axion mass). For plotting purposes, we fixed $\tan \beta=10$ for the base models and their extensions. (Results for other large values of $\tan \beta$ would not be visually distinguishable.) In DFSZ-I and the base models and their extensions, for a fixed $\tan \beta$, horizontal lines in these plots have constant $|N|$, while vertical lines have constant $E / N$. The horizontal dotted line in the top panel corresponds to $|N|=3$ for fixed $\tan \beta=10$ (in DFSZ-Itype models), the vertical dotted line corresponds to $E / N=8 / 3$, and the intersection point at $(1,1)$, of course, corresponds to the benchmark DFSZ-I with $\tan \beta=10$. In the top-right corner of the plot in the top panel, the horizontal dashed lines correspond to cases with $N_{\mathrm{DW}}=$ 1 in the extensions of base models with $|N|=1 / 6$ and $|N|=1 / 2$ for a fixed $\tan \beta=10$. As noted earlier, one or more cases with $N_{\mathrm{DW}}=1$ provide for the largest axionphoton coupling [when $E / N$ is far away from 1.92(4)] and axion-electron coupling (for smallest $|N|$ when tree-level contributions dominate), as is reflected in the figure. In the bottom panel, there are additional points on the horizontal line with $|N|=1 / 2$ that are not present in the top panel; these points are from the extensions of base model $\mathrm{B}_{\text {III }}$ with $|N|=1 / 2$ and $N_{\text {DW }}=3$. Also, in the figure, the DFSZ-I and DFSZ-II benchmark models are shown for various values of $\tan \beta$. We can therefore understand the impact of $\tan \beta$ on the points for base models and its extensions, as they have a similar $\tan \beta$ dependence as that of DFSZ-I.

Finally, we turn to the axion-nucleon couplings, for which the neutron coupling is most accessible to constraint. Figure 5 shows the axion-neutron coupling as a function of the axion mass $m_{A}$ and the decay constant $f_{A}$, choosing $\tan \beta=10$ for plotting purposes. Because of significant uncertainties in the axion coupling predictions, each case in the figure is a shaded band instead of a line. First, bands are 
shown for the $N_{\text {DW }}=1$ cases that occur in $N= \pm 1 / 2$ and $\pm 1 / 6$ extensions of the base models. The axion-nucleon coupling, in general, depends on the sign of $N$, but in the case of the neutron the shaded bands for $N=1 / 2$ and $N=1 / 6$ very nearly overlap with the shaded bands for $N=-1 / 2$ and $N=-1 / 6$, respectively, so we have combined them. Also shown is the band of maximum allowed axion-neutron coupling in the extensions with only a $\mathbf{5}+\overline{\mathbf{5}}$, which occurs for $N=3 / 2$ in base model $\mathrm{B}_{\mathrm{I}}$ extended with a $\mathbf{5}+\overline{\mathbf{5}}$ at $M_{\text {int }}$. In addition, ranges are shown for the supersymmetric base models and the DFSZ and KSVZ models, as labeled. Note that, for a fixed $\tan \beta$ and $N$, the base models and DFSZ-I and DFSZ-II all have the same axion-nucleon couplings. Within uncertainties, the KSVZ axion can accidentally decouple from the neutron, so the shading in that case extends to arbitrarily low $g_{A n}$.

The existence of the neutrino signal from supernova SN 1987A has been interpreted by Ref. [120] to put the following constraint on axion-nucleon couplings:

$$
g_{A n}^{2}+0.61 g_{A p}^{2}+0.53 g_{A n} g_{A p} \lesssim 8.26 \times 10^{-19} .
$$

However, it has been suggested, e.g., in Ref. [121], that such bounds should be taken as a guide rather than a sharp bound, given the uncertainties involved. Taken at face value, the bound implies, for large $\tan \beta$,

$$
f_{A} \gtrsim \sqrt{0.15+0.66 / N^{2}}\left(10^{9} \mathrm{GeV}\right),
$$

for our models, by using Eqs. (2.51) and (2.52). (There is also a term proportional to $1 / N$ under the square root, but its coefficient is consistent with 0 within uncertainties.) For the critical case of small $|N|$, this is not quite as strong as Eq. (4.1) obtained above from the constraints on $g_{A e}$ from red-giant and white dwarf cooling. In Fig. 5, we show the bound as it applies to the supersymmetric DFSZ models as parameterized by Eqs. (2.51) and (2.52) with large $\tan \beta$, recognizing that it is not a model-independent bound on $g_{A n}$ (because other models can have different correlations between $g_{A n}$ and $g_{A p}$ ) and in any case may not be robust in detail.

There is also an even stronger candidate bound [122] of $\left|g_{A n}\right|<2.8 \times 10^{-10}$ from avoiding too much cooling of the hot neutron star HESS J1731-347. However, since it is difficult to account for the temperature of this particular object even without axionic cooling [123], we do not include this bound in Fig. 5.

Also shown in Fig. 5 are the experimental reach for the axion-neutron coupling of phase II of the proposed CASPEr wind experiment [124] and a projection for the ARIADNE experiment $[125,126]$. ARIADNE would be sensitive to the product of $g_{A n}$ and the $C P$-odd scalar coupling $g_{A n}^{S}$ (as in $\mathcal{L}^{C P \text {-odd }} \supset-A g_{A n}^{s} \bar{\Psi}_{n} \Psi_{n}$ ), and the region shown in Fig. 5 is based on an optimistic choice of $g_{A n}^{s}=10^{-12} \mathrm{GeV} / f_{A} \quad[20,125,127]$. The coupling $g_{A n}^{s}$ could arise from a small nonzero $C P$ violation in $\theta_{\text {eff }}$ and so cannot be uniquely predicted by the other relevant model parameters $N$ and $\tan \beta$. We note that, at least with this optimistic assumption, ARIADNE can probe $g_{A n}$ for $f_{A}$ up to about $2.5 \times 10^{10} \mathrm{GeV}$ for the supersymmetric DFSZ base models, which are invisible to the projected searches for $g_{A \gamma}$.

\section{CONCLUSION}

In this paper, we considered supersymmetric DFSZ-type axion models with the field content of the MSSM plus two gauge-singlet fields $X$ and $Y$ that spontaneously break the PQ symmetry and some extra vectorlike quark and lepton supermultiplets. These models simultaneously give a solution to the $\mu$ problem and the strong $C P$ problem. The extra vectorlike content is chosen such that the perturbative gauge coupling unification is maintained. The PQ symmetry is spontaneously broken by the scalar components of $X$ and $Y$, which acquire intermediate-scale vacuum expectation values, simultaneously giving rise to a high-quality nearly invisible axion with a decay constant within the current astrophysical limits and a $\mu$ term around the $\mathrm{TeV}$ scale. The axino and saxion also have masses of the order of the TeV scale. Different combinations of mass terms for the additional vectorlike supermultiplets involving $X$ and $Y$ result in different combinations of the PQ anomaly coefficients $(N, E)$, which, in turn, determines the low-energy axion couplings.

For the base models and their extensions, we studied how to obtain the PQ symmetry as an accidental symmetry that is guarded against the dangerous PQ-violating superpotential terms of the form $X^{j} Y^{p-j} / M_{P}^{p-3}$ to an extent that is compatible with the experimental constraint on the QCD $\theta$ parameter, by imposing anomaly-free discrete non- $R$ or $R$ $Z_{n}$ symmetries with or without the Green-Schwarz mechanism. If the axion decay constant $f_{A}$ is as low as $10^{9} \mathrm{GeV}$, we can allow $p=7$, and for $f_{A} \lesssim 10^{12} \mathrm{GeV}$, we may instead need a higher suppression of up to $p=12$. In order for the $Z_{n}^{R}$ symmetries (which reduce to non- $R Z_{n}$ symmetry if the $Z_{n}^{R}$ charge of the gauginos $r=0$ ) to be anomaly-free, we impose a weaker constraint along with the additional stronger constraint on the $Z_{n}^{R} \times G \times G$ anomalies for $G=S U(3)_{c}, S U(2)_{L}$, and $U(1)_{Y}$ as discussed in Sec. III. Out of all possible non- $R Z_{n}$ symmetries for the base models, the only cases with adequate suppression $(p \geq 7)$ that satisfy the stronger set of anomaly constraints are $Z_{36}$ for base model $\mathrm{B}_{\text {III }}$ with $p=12$ and $Z_{36}$ for $\mathrm{B}_{\mathrm{IV}}$ with $p=8$. Both of these cases require the GreenSchwarz mechanism. On the other hand, there are a lot more anomaly-free $Z_{n} R$ symmetries for each base model, some of which do not require the Green-Schwarz mechanism. For example, there is a $Z_{54}^{R}$ for base model $\mathrm{B}_{\text {III }}$ with $p=10$ and $Z_{12}^{R}$ for base model $\mathrm{B}_{\mathrm{IV}}$ with only $p=7$, both of which do not require the Green-Schwarz mechanism. 
With the Green-Schwarz mechanism, there are many more $Z_{n}^{R}$ symmetries that have lower $n$ and higher suppression $p$. In our approach, the discrete symmetry is fundamental and exact (being anomaly-free) and the PQ symmetry is not fundamental and approximate (being an accidental consequence of the discrete symmetry).

Adding vectorlike supermultiplets adds greatly to the possibilities for both discrete non- $R$ and $R$ symmetries. For each possible pair of PQ anomaly coefficients $(N, E)$ in the extensions, we find that there are always anomaly-free discrete symmetries that protect the PQ symmetry to a high degree of accuracy. Not only do these discrete symmetries provide for an accidental $U(1)_{\mathrm{PQ}}$ symmetry, but they also can forbid dangerous baryon-number- and lepton-number-violating operators, in most cases, that could mediate dangerous proton decay. There are so many available discrete symmetries that we did not attempt a complete categorization. The additional fields and the discrete symmetry of the models we have proposed increase the complexity of the MSSM. The discrete symmetry could arise from an additional $U(1)$ symmetry in the ultraviolet, but other than that we do not have any insight to offer as to the reasons for this additional complexity, other than the problems that it solves.

In our models, the vectorlike supermultiplets are coupled to Standard Model quark and lepton superfields to avoid cosmological problems. These couplings could be very small in magnitude while still allowing the vectorlike particles to decay promptly. Thus, while they would, in general, include additional $C P$-violating phases, the resulting $C P$-violating effects on the Standard Model could easily be negligible if the magnitudes of the couplings are sufficiently small. We also note that in the MSSM there are potential $C P$-violating effects from gaugino masses and the $\mu$ parameter. Our framework has nothing to say about these effects, except that they are not worse than in the usual MSSM, and as usual they are ameliorated for superpartners in the $\mathrm{TeV}$ range.

Because of the soft supersymmetry-breaking terms, the scalar components of the vectorlike chiral supermultiplets tend to be heavier than their corresponding fermions, as studied, for example, in Ref. [128]. Therefore, the constraints on the masses of the extra vectorlike supermultiplets come from the searches for pair production of vectorlike quarks and leptons at the particle colliders. From the most recent LHC searches by the ATLAS and CMS Collaborations for vectorlike quarks $[129,130]$, the up-type (down-type) vectorlike quarks that are assumed to decay to the top (bottom) quarks are excluded at 95\% confidence level up to $1310-1600 \mathrm{GeV}$ (1200-1570 GeV), depending on its branching ratios. And, the weak isodoublet vectorlike leptons that decay to the tau leptons are excluded at $95 \%$ confidence level from 120 to $790 \mathrm{GeV}$ by the CMS Collaboration in Ref. [131]. Lastly, as was pointed out in Refs. [132,133], the weak isosinglet charged vectorlike leptons that mix with the tau lepton have essentially no reach prospects at the current and the future proton-proton colliders due to low pair-production cross sections and unfavorable branching ratios.

After the PQ breaking, the axion potential typically acquires more than one inequivalent degenerate minimum, given by the domain wall number $N_{\text {DW }}$, leading to a cosmological domain wall problem if the symmetry is broken in the postinflationary era. Models with $N_{\text {DW }}=1$ evade this problem. The four base models without extra vectorlike supermultiplets have $N_{\mathrm{DW}} \neq 1$ and, therefore, may suffer from the domain wall problem. They also have suppressed axion-photon couplings compared to the standard benchmark QCD axion models and so may be invisible to future proposed direct axion searches. However, in the extensions of base models that include extra vectorlike supermultiplets, we obtained a wide variety of larger axion couplings. It is notable that the extensions with $N_{\mathrm{DW}}=1$, which always have at least one strongly interacting vectorlike supermultiplet at the intermediate scale, have the smallest $|N|$ and, therefore, give rise to enhanced axion couplings, which are likely to be within reach of future axion searches.

\section{ACKNOWLEDGMENTS}

This work is supported in part by the National Science Foundation under Grant No. 2013340.
[1] C. Abel et al. (nEDM Collaboration), Measurement of the Permanent Electric Dipole Moment of the Neutron, Phys. Rev. Lett. 124, 081803 (2020).

[2] R. D. Peccei and H. R. Quinn, CP Conservation in the Presence of Instantons, Phys. Rev. Lett. 38, 1440 (1977).

[3] R. D. Peccei and H. R. Quinn, Constraints imposed by $C P$ conservation in the presence of instantons, Phys. Rev. D 16, 1791 (1977).

[4] S. Weinberg, A New Light Boson?, Phys. Rev. Lett. 40, 223 (1978).
[5] F. Wilczek, Problem of Strong $P$ and $T$ Invariance in the Presence of Instantons, Phys. Rev. Lett. 40, 279 (1978).

[6] J.E. Kim, Weak Interaction Singlet and Strong $C P$ Invariance, Phys. Rev. Lett. 43, 103 (1979).

[7] M. A. Shifman, A. I. Vainshtein, and V. I. Zakharov, Can confinement ensure natural $C P$ invariance of strong interactions?, Nucl. Phys. B166, 493 (1980).

[8] M. Dine, W. Fischler, and M. Srednicki, A simple solution to the strong $C P$ problem with a harmless axion, Phys. Lett. 104B, 199 (1981). 
[9] A. R. Zhitnitsky, On possible suppression of the axion hadron interactions (in Russian), Sov. J. Nucl. Phys. 31, 260 (1980).

[10] J. Preskill, M. B. Wise, and F. Wilczek, Cosmology of the invisible axion, Phys. Lett. 120B, 127 (1983).

[11] L. F. Abbott and P. Sikivie, A cosmological bound on the invisible axion, Phys. Lett. 120B, 133 (1983).

[12] M. Dine and W. Fischler, The not so harmless axion, Phys. Lett. 120B, 137 (1983).

[13] M. S. Turner, Windows on the axion, Phys. Rep. 197, 67 (1990).

[14] P. Sikivie, The pool table analogy to axion physics, Phys. Today 49, No. 12, 22 (1996).

[15] M. Dine, TASI lectures on the strong $C P$ problem, arXiv: hep-ph/0011376.

[16] R. D. Peccei, The strong $C P$ problem and axions, Lect. Notes Phys. 741, 3 (2008).

[17] J.E. Kim and G. Carosi, Axions and the strong $C P$ problem, Rev. Mod. Phys. 82, 557 (2010); 91, 049902 (E) (2019).

[18] D. J. E. Marsh, Axion cosmology, Phys. Rep. 643, 1 (2016).

[19] A. Hook, TASI lectures on the strong $C P$ problem and axions, Proc. Sci., TASI2018 (2019) 004 [arXiv: 1812.02669].

[20] L. Di Luzio, M. Giannotti, E. Nardi, and L. Visinelli, The landscape of QCD axion models, Phys. Rep. 870, 1 (2020).

[21] P. Sikivie, Invisible axion search methods, Rev. Mod. Phys. 93, 015004 (2021).

[22] Y. K. Semertzidis and S. Youn, Axion dark matter: How to detect it?, arXiv:2104.14831.

[23] S. P. Martin, A supersymmetry primer, Adv. Ser. Dir. High Energy Phys. 18, 1 (1998).

[24] J. E. Kim and H. P. Nilles, The $\mu$-problem and the strong CP-problem, Phys. Lett. 138B, 150 (1984).

[25] H. Murayama, H. Suzuki, and T. Yanagida, Radiative breaking of Peccei-Quinn symmetry at the intermediate mass scale, Phys. Lett. B 291, 418 (1992).

[26] S. P. Martin, Dimensionless supersymmetry breaking couplings, flat directions, and the origin of intermediate mass scales, Phys. Rev. D 61, 035004 (2000).

[27] S. P. Martin, Collider signals from slow decays in supersymmetric models with an intermediate scale solution to the $\mu$ problem, Phys. Rev. D 62, 095008 (2000).

[28] K. Choi, E. J. Chun, and J. E. Kim, Cosmological implications of radiatively generated axion scale, Phys. Lett. B 403, 209 (1997).

[29] K. J. Bae, H. Baer, V. Barger, and D. Sengupta, Revisiting the SUSY $\mu$ problem and its solutions in the LHC era, Phys. Rev. D 99, 115027 (2019).

[30] H. M. Georgi, L. J. Hall, and M. B. Wise, Grand unified models with an automatic Peccei-Quinn symmetry, Nucl. Phys. B192, 409 (1981).

[31] M. Dine and N. Seiberg, String theory and the strong $C P$ problem, Nucl. Phys. B273, 109 (1986).

[32] S. M. Barr and D. Seckel, Planck scale corrections to axion models, Phys. Rev. D 46, 539 (1992).

[33] M. Kamionkowski and J. March-Russell, Planck scale physics and the Peccei-Quinn mechanism, Phys. Lett. B 282, 137 (1992).
[34] R. Holman, S. D. H. Hsu, T. W. Kephart, E. W. Kolb, R. Watkins, and L. M. Widrow, Solutions to the strong-CP problem in a world with gravity, Phys. Lett. B 282, 132 (1992).

[35] J. E. Kim, A composite invisible axion, Phys. Rev. D 31, 1733 (1985).

[36] K. Choi and J. E. Kim, Dynamical axion, Phys. Rev. D 32, 1828 (1985).

[37] L. Randall, Composite axion models and Planck scale physics, Phys. Lett. B 284, 77. (1992).

[38] M. Redi and R. Sato, Composite accidental axions, J. High Energy Phys. 05 (2016) 104.

[39] L. Di Luzio, E. Nardi, and L. Ubaldi, Accidental PecceiQuinn Symmetry Protected to Arbitrary Order, Phys. Rev. Lett. 119, 011801 (2017).

[40] B. Lillard and T. M. P. Tait, A composite axion from a supersymmetric product group, J. High Energy Phys. 11 (2017) 005.

[41] B. Lillard and T. M. P. Tait, A high quality composite axion, J. High Energy Phys. 11 (2018) 199.

[42] H. S. Lee and W. Yin, Peccei-Quinn symmetry from a hidden gauge group structure, Phys. Rev. D 99, 015041 (2019).

[43] M. B. Gavela, M. Ibe, P. Quilez, and T. T. Yanagida, Automatic Peccei-Quinn symmetry, Eur. Phys. J. C 79, 542 (2019).

[44] M. Ardu, L. Di Luzio, G. Landini, A. Strumia, D. Teresi, and J. W. Wang, Axion quality from the (anti)symmetric of $\mathrm{SU}(\mathcal{N})$, J. High Energy Phys. 11 (2020) 090.

[45] W. Yin, Scale and quality of Peccei-Quinn symmetry and weak gravity conjectures, J. High Energy Phys. 10 (2020) 032.

[46] H. C. Cheng and D. E. Kaplan, Axions and a gauged Peccei-Quinn symmetry, arXiv:hep-ph/0103346.

[47] C. T. Hill and A. K. Leibovich, Natural theories of ultralow mass PNGB's: Axions and quintessence, Phys. Rev. D 66, 075010 (2002); L. Di Luzio, Accidental SO(10) axion from gauged flavour, J. High Energy Phys. 11 (2020) 074.

[48] H. Fukuda, M. Ibe, M. Suzuki, and T. T. Yanagida, A "gauged" $U(1)$ Peccei-Quinn symmetry, Phys. Lett. B 771, 327 (2017).

[49] M. Duerr, K. Schmidt-Hoberg, and J. Unwin, Protecting the axion with local baryon number, Phys. Lett. B 780, 553 (2018).

[50] H. Fukuda, M. Ibe, M. Suzuki, and T. T. Yanagida, Gauged Peccei-Quinn symmetry - a case of simultaneous breaking of SUSY and PQ symmetry, J. High Energy Phys. 07 (2018) 128.

[51] M. Ibe, M. Suzuki, and T. T. Yanagida, $B-L$ as a gauged Peccei-Quinn symmetry, J. High Energy Phys. 08 (2018) 049.

[52] G. Choi, M. Suzuki, and T. T. Yanagida, QCD axion from a spontaneously broken $B-L$ gauge symmetry, J. High Energy Phys. 07 (2020) 048.

[53] N. Chen, Y. Liu, and Z. Teng, An axion model with the SU(6) unification, arXiv:2106.00223.

[54] A. G. Dias, V. Pleitez, and M. D. Tonasse, Naturally light invisible axion in models with large local discrete symmetries, Phys. Rev. D 67, 095008 (2003). 
[55] A. G. Dias, C. A. de S. Pires, and P. S. Rodrigues da Silva, Discrete symmetries, invisible axion and lepton number symmetry in an economic 3-3-1 model, Phys. Rev. D 68, 115009 (2003).

[56] L. M. Carpenter, M. Dine, and G. Festuccia, Dynamics of the Peccei Quinn scale, Phys. Rev. D 80, 125017 (2009).

[57] A. Ringwald and K. Saikawa, Axion dark matter in the post-inflationary Peccei-Quinn symmetry breaking scenario, Phys. Rev. D 93, 085031 (2016).

[58] F. Björkeroth, E. J. Chun, and S. F. King, Accidental Peccei-Quinn symmetry from discrete flavour symmetry and Pati-Salam, Phys. Lett. B 777, 428 (2018).

[59] E. J. Chun and A. Lukas, Discrete gauge symmetries in axionic extensions of the SSM, Phys. Lett. B 297, 298 (1992).

[60] K. S. Babu, I. Gogoladze, and K. Wang, Stabilizing the axion by discrete gauge symmetries, Phys. Lett. B 560, 214 (2003).

[61] H. M. Lee, S. Raby, M. Ratz, G. G. Ross, R. Schieren, K. Schmidt-Hoberg, and P. K. S. Vaudrevange, Discrete $R$ symmetries for the MSSM and its singlet extensions, Nucl. Phys. B850, 1 (2011).

[62] K. Harigaya, M. Ibe, K. Schmitz, and T. T. Yanagida, Peccei-Quinn symmetry from a gauged discrete $R$ symmetry, Phys. Rev. D 88, 075022 (2013).

[63] K. Harigaya, M. Ibe, K. Schmitz, and T. T. Yanagida, Peccei-Quinn symmetry from dynamical supersymmetry breaking, Phys. Rev. D 92, 075003 (2015).

[64] H. Baer, V. Barger, and D. Sengupta, Gravity safe, electroweak natural axionic solution to strong $C P$ and SUSY $\mu$ problems, Phys. Lett. B 790, 58 (2019); H. Baer, V. Barger, D. Sengupta, H. Serce, K. Sinha, and R. W. Deal, Is the magnitude of the Peccei-Quinn scale set by the landscape?, Eur. Phys. J. C 79, 897 (2019).

[65] J. Kawamura and S. Raby, Qualities of the axion and LSP in Pati-Salam unification with $\mathbb{Z}_{4}^{R} \times \mathbb{Z}_{N}$ symmetry, Phys. Rev. D 103, 015002 (2021).

[66] Y. Nakai and M. Suzuki, Axion quality from superconformal dynamics, Phys. Lett. B 816, 136239 (2021).

[67] H. Georgi and S. L. Glashow, Unity of All Elementary Particle Forces, Phys. Rev. Lett. 32, 438 (1974).

[68] S. Borsanyi, Z. Fodor, J. Guenther, K. H. Kampert, S. D. Katz, T. Kawanai, T. G. Kovacs, S. W. Mages, A. Pasztor, F. Pittler et al., Calculation of the axion mass based on high-temperature lattice quantum chromodynamics, Nature (London) 539, 69 (2016).

[69] G. Grilli di Cortona, E. Hardy, J. P. Vega, and G. Villadoro, The QCD axion, precisely, J. High Energy Phys. 01 (2016) 034.

[70] M. Srednicki, Axion couplings to matter: 1. CP-conserving parts, Nucl. Phys. B260, 689 (1985).

[71] S. Chang and K. Choi, Hadronic axion window and the big bang nucleosynthesis, Phys. Lett. B 316, 51 (1993).

[72] K. Choi, S. H. Im, C. B. Park, and S. Yun, Minimal flavor violation with axion-like particles, J. High Energy Phys. 11 (2017) 070.

[73] M. Chala, G. Guedes, M. Ramos, and J. Santiago, Running in the ALPs, Eur. Phys. J. C 81, 181 (2021).
[74] M. Bauer, M. Neubert, S. Renner, M. Schnubel, and A. Thamm, The low-energy effective theory of axions and ALPs, J. High Energy Phys. 04 (2021) 063.

[75] K. Choi, S. H. Im, H. J. Kim, and H. Seong, Precision axion physics with running axion couplings, arXiv: 2106.05816.

[76] P. Sikivie, Of Axions, Domain Walls and the Early Universe, Phys. Rev. Lett. 48, 1156 (1982).

[77] A. Ernst, A. Ringwald, and C. Tamarit, Axion predictions in $S O(10) \times U(1)_{\mathrm{PQ}}$ models, J. High Energy Phys. 02 (2018) 103.

[78] G. Lazarides and Q. Shafi, Axion models with no domain wall problem, Phys. Lett. B 115, 21 (1982).

[79] A. Vilenkin and A. E. Everett, Cosmic Strings and Domain Walls in Models with Goldstone and PseudoGoldstone Bosons, Phys. Rev. Lett. 48, 1867 (1982).

[80] H. Georgi and M. B. Wise, Hiding the invisible axion, Phys. Lett. 116B, 123 (1982).

[81] L. E. Ibanez and G. G. Ross, Discrete gauge symmetry anomalies, Phys. Lett. B 260, 291 (1991).

[82] L. E. Ibanez and G. G. Ross, Discrete gauge symmetries and the origin of baryon and lepton number conservation in supersymmetric versions of the standard model, Nucl. Phys. B368, 3 (1992).

[83] T. Banks and M. Dine, Note on discrete gauge anomalies, Phys. Rev. D 45, 1424 (1992).

[84] L. E. Ibanez, More about discrete gauge anomalies, Nucl. Phys. B398, 301 (1993).

[85] L. M. Krauss and F. Wilczek, Discrete Gauge Symmetry in Continuum Theories, Phys. Rev. Lett. 62, 1221 (1989).

[86] M. B. Green and J. H. Schwarz, Anomaly cancellation in supersymmetric $D=10$ gauge theory and superstring theory, Phys. Lett. 149B, 117 (1984).

[87] J. C. Pati and A. Salam, Lepton number as the fourth color, Phys. Rev. D 10, 275 (1974); 11, 703(E) (1975).

[88] G. R. Farrar and P. Fayet, Phenomenology of the production, decay, and detection of new hadronic states associated with supersymmetry, Phys. Lett. 76B, 575 (1978).

[89] S. Dimopoulos and H. Georgi, Softly broken supersymmetry and SU(5), Nucl. Phys. B193, 150 (1981).

[90] S. Weinberg, Supersymmetry at ordinary energies. 1. Masses and conservation laws, Phys. Rev. D 26, 287 (1982).

[91] K. J. Bae, H. Baer, and H. Serce, Prospects for axion detection in natural SUSY with mixed axion-higgsino dark matter: back to invisible?, J. Cosmol. Astropart. Phys. 06 (2017) 024.

[92] D. S. Akerib et al. (LUX Collaboration), First Searches for Axions and Axionlike Particles with the LUX Experiment, Phys. Rev. Lett. 118, 261301 (2017).

[93] F. Capozzi and G. Raffelt, Axion and neutrino bounds improved with new calibrations of the tip of the red-giant branch using geometric distance determinations, Phys. Rev. D 102, 083007 (2020).

[94] O. Straniero, C. Pallanca, E. Dalessandro, I. Dominguez, F. R. Ferraro, M. Giannotti, A. Mirizzi, and L. Piersanti, The RGB tip of galactic globular clusters and the revision of the axion-electron coupling bound, Astron. Astrophys. 644, A166 (2020). 
[95] J. Isern, E. Garcia-Berro, S. Torres, and S. Catalan, Axions and the cooling of white dwarf stars, Astrophys. J. Lett. 682, L109 (2008).

[96] J. Isern, S. Catalan, E. Garcia-Berro, and S. Torres, Axions and the white dwarf luminosity function, J. Phys. Conf. Ser. 172, 012005 (2009).

[97] M. M. M. Bertolami, B. E. Melendez, L. G. Althaus, and J. Isern, Revisiting the axion bounds from the Galactic white dwarf luminosity function, J. Cosmol. Astropart. Phys. 10 (2014) 069.

[98] M. Giannotti, I. G. Irastorza, J. Redondo, A. Ringwald, and K. Saikawa, Stellar recipes for axion hunters, J. Cosmol. Astropart. Phys. 10 (2017) 010.

[99] S. Andriamonje et al. (CAST Collaboration), An improved limit on the axion-photon coupling from the CAST experiment, J. Cosmol. Astropart. Phys. 04 (2007) 010.

[100] V. Anastassopoulos et al. (CAST Collaboration), New CAST limit on the axion-photon interaction, Nat. Phys. 13, 584 (2017).

[101] A. Ayala, I. Domínguez, M. Giannotti, A. Mirizzi, and O. Straniero, Revisiting the Bound on Axion-Photon Coupling from Globular Clusters, Phys. Rev. Lett. 113, 191302 (2014).

[102] C. Bartram et al. (ADMX Collaboration), Axion dark matter experiment: Run 1B analysis details, Phys. Rev. D 103, 032002 (2021).

[103] C. Boutan et al. (ADMX Collaboration), Piezoelectrically Tuned Multimode Cavity Search for Axion Dark Matter, Phys. Rev. Lett. 121, 261302 (2018).

[104] N. Du et al. (ADMX Collaboration), A Search for Invisible Axion Dark Matter with the Axion Dark Matter Experiment, Phys. Rev. Lett. 120, 151301 (2018).

[105] S. J. Asztalos et al. (ADMX Collaboration), A SQUIDBased Microwave Cavity Search for Dark-Matter Axions, Phys. Rev. Lett. 104, 041301 (2010).

[106] S. Lee, S. Ahn, J. Choi, B. R. Ko, and Y. K. Semertzidis, Axion Dark Matter Search around 6.7 $\mu \mathrm{eV}$, Phys. Rev. Lett. 124, 101802 (2020).

[107] S. De Panfilis, A. C. Melissinos, B. E. Moskowitz, J. T. Rogers, Y. K. Semertzidis, W. Wuensch, H. J. Halama, A. G. Prodell, W. B. Fowler, and F. A. Nezrick, Limits on the Abundance and Coupling of Cosmic Axions at $4.5<m_{a}<5.0 \mu \mathrm{eV}$, Phys. Rev. Lett. 59, 839 (1987).

[108] C. Hagmann, P. Sikivie, N. S. Sullivan, and D. B. Tanner, Results from a search for cosmic axions, Phys. Rev. D 42, 1297 (1990).

[109] K. M. Backes et al. (HAYSTAC Collaboration), A quantumenhanced search for dark matter axions, Nature (London) 590, 238 (2021).

[110] L. Zhong et al. (HAYSTAC Collaboration), Results from phase 1 of the HAYSTAC microwave cavity axion experiment, Phys. Rev. D 97, 092001 (2018).

[111] D. Alesini, C. Braggio, G. Carugno, N. Crescini, D. D’Agostino, D. Di Gioacchino, R. Di Vora, P. Falferi, S. Gallo, U. Gambardella et al., Galactic axions search with a superconducting resonant cavity, Phys. Rev. D 99, 101101 (2019).

[112] B. T. McAllister, G. Flower, E. N. Ivanov, M. Goryachev, J. Bourhill, and M. E. Tobar, The ORGAN experiment: An axion haloscope above $15 \mathrm{GHz}$, Phys. Dark Universe 18, 67 (2017).

[113] C. O'Hare, cajohare/AxionLimits: AxionLimits (Version v1.0). Zenodo (2020) https://doi.org/10.5281/zenodo .3932430 .

[114] I. Shilon, A. Dudarev, H. Silva, and H. H. J. ten Kate, Conceptual design of a new large superconducting toroid for IAXO, the New International AXion Observatory, IEEE Trans. Appl. Supercond. 23, 4500604 (2013).

[115] I. Stern, ADMX status, Proc. Sci., ICHEP2016 (2016) 198 [arXiv:1612.08296].

[116] D. Alesini, D. Babusci, D. Di Gioacchino, C. Gatti, G. Lamanna, and C. Ligi, The KLASH proposal, arXiv: 1707.06010.

[117] S. Beurthey, N. Böhmer, P. Brun, A. Caldwell, L. Chevalier, C. Diaconu, G. Dvali, P. Freire, E. Garutti, C. Gooch et al., MADMAX status report, arXiv:2003.10894.

[118] M. Lawson, A. J. Millar, M. Pancaldi, E. Vitagliano, and F. Wilczek, Tunable Axion Plasma Haloscopes, Phys. Rev. Lett. 123, 141802 (2019).

[119] J. Schütte-Engel, D. J. E. Marsh, A. J. Millar, A. Sekine, F. Chadha-Day, S. Hoof, M. Ali, K. C. Fong, E. Hardy, and L. Šmejkal, Axion quasiparticles for axion dark matter detection, arXiv:2102.05366.

[120] P. Carenza, T. Fischer, M. Giannotti, G. Guo, G. MartínezPinedo, and A. Mirizzi, Improved axion emissivity from a supernova via nucleon-nucleon Bremsstrahlung, J. Cosmol. Astropart. Phys. 10 (2019) 016; 05 (2020) 01(E).

[121] T. Fischer, S. Chakraborty, M. Giannotti, A. Mirizzi, A. Payez, and A. Ringwald, Probing axions with the neutrino signal from the next galactic supernova, Phys. Rev. D 94, 085012 (2016).

[122] M. V. Beznogov, E. Rrapaj, D. Page, and S. Reddy, Constraints on axion-like particles and nucleon pairing in dense matter from the hot neutron star in HESS J1731347, Phys. Rev. C 98, 035802 (2018).

[123] A. Sedrakian, Axion cooling of neutron stars. II. Beyond hadronic axions, Phys. Rev. D 99, 043011 (2019).

[124] D. F. J. Kimball, S. Afach, D. Aybas, J. W. Blanchard, D. Budker, G. Centers, M. Engler, N. L. Figueroa, A. Garcon, and P.W. Graham, Overview of the cosmic axion spin precession experiment (CASPEr), Springer Proc. Phys. 245, 105 (2020).

[125] A. Arvanitaki and A. A. Geraci, Resonantly Detecting Axion-Mediated Forces with Nuclear Magnetic Resonance, Phys. Rev. Lett. 113, 161801 (2014).

[126] A. A. Geraci et al. (ARIADNE Collaboration), Progress on the ARIADNE axion experiment, Springer Proc. Phys. 211, 151 (2018).

[127] C. A. J. O'Hare and E. Vitagliano, Cornering the axion with $C P$-violating interactions, Phys. Rev. D 102, 115026 (2020).

[128] S. P. Martin, Extra vector-like matter and the lightest Higgs scalar boson mass in low-energy supersymmetry, Phys. Rev. D 81, 035004 (2010).

[129] A. M. Sirunyan et al. (CMS Collaboration), A search for bottom-type, vectorlike quark pair production in a fully hadronic final state in proton-proton collisions at $\sqrt{s}=13$ TeV, Phys. Rev. D 102, 112004 (2020); Search for pair production of vectorlike quarks in the fully 
hadronic final state, Phys. Rev. D 100, 072001 (2019); Search for vector-like quarks in events with two oppositely charged leptons and jets in proton-proton collisions at $\sqrt{s}=13 \mathrm{TeV}$, Eur. Phys. J. C 79, 364 (2019); Search for vector-like $\mathrm{T}$ and $\mathrm{B}$ quark pairs in final states with leptons at $\sqrt{s}=13 \mathrm{TeV}$, J. High Energy Phys. 08 (2018) 177.

[130] M. Aaboud et al. (ATLAS Collaboration), Search for pairproduction of vector-like quarks in $p p$ collision events at $\sqrt{s}=13 \mathrm{TeV}$ with at least one leptonically-decaying $Z$ boson and a third-generation quark with the ATLAS detector, Report No. ATLAS-CONF-2021-024; Combination of the Searches for Pair-Produced Vectorlike Partners of the Third-Generation Quarks at $\sqrt{s}=13 \mathrm{TeV}$ with the ATLAS Detector, Phys. Rev. Lett. 121, 211801 (2018); Search for pair production of heavy vectorlike quarks decaying into hadronic final states in $p p$ collisions at $\sqrt{s}=13 \mathrm{TeV}$ with the ATLAS detector, Phys. Rev. D 98, 092005 (2018); Search for pair- and single-production of vectorlike quarks in final states with at least one $Z$ boson decaying into a pair of electrons or muons in $p p$ collision data collected with the ATLAS detector at $\sqrt{s}=13 \mathrm{TeV}$, Phys. Rev. D 98, 112010 (2018); Search for pair production of heavy vector-like quarks decaying into high- $p_{T} W$ bosons and top quarks in the lepton-plus-jets final state in $p p$ collisions at $\sqrt{s}=13 \mathrm{TeV}$ with the ATLAS detector, J. High Energy Phys. 08 (2018) 048; Search for pair production of up-type vector-like quarks and for four-topquark events in final states with multiple $b$-jets with the ATLAS detector, J. High Energy Phys. 07 (2018) 089.

[131] A. M. Sirunyan et al. (CMS Collaboration), Search for vectorlike leptons in multilepton final states in protonproton collisions at $\sqrt{s}=13 \mathrm{TeV}$, Phys. Rev. D 100, 052003 (2019).

[132] N. Kumar and S. P. Martin, Vectorlike leptons at the large hadron collider, Phys. Rev. D 92, 115018 (2015).

[133] P. N. Bhattiprolu and S. P. Martin, Prospects for vectorlike leptons at future proton-proton colliders, Phys. Rev. D 100, 015033 (2019). 